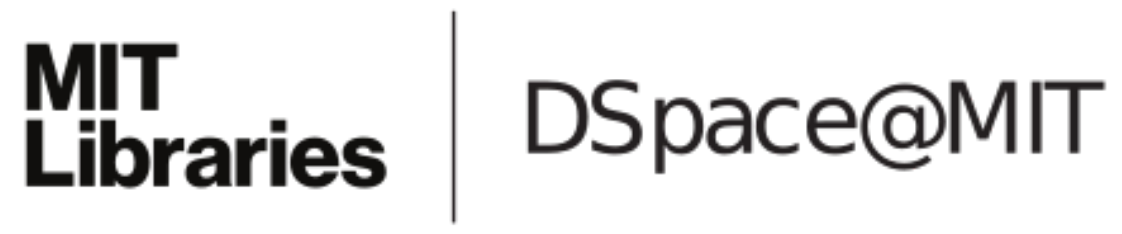

\author{
MIT Open Access Articles
}

A budget for the size of convective self\#aggregation

The MIT Faculty has made this article openly available. Please share how this access benefits you. Your story matters.

Citation: Beucler, Tom; Cronin, Timothy. "A budget for the size of convective self\#aggregation." Quarterly Journal of the Royal Meteorological Society 145 (2019): 947-966. https:// doi.org/10.1002/qj.3468

As Published: $10.1002 /$ QJ.3468

Publisher: Wiley

Persistent URL: https://hdl.handle.net/1721.1/126099

Version: Author's final manuscript: final author's manuscript post peer review, without publisher's formatting or copy editing

Terms of use: Creative Commons Attribution-Noncommercial-Share Alike 


\section{A Budget for the Size of Convective Self-aggregation}

\section{Tom Beucler $^{1}$ | Timothy Cronin ${ }^{1}$}

\author{
${ }^{1}$ Lorenz Center, Department of Earth \\ Atmospheric and Planetary Sciences, \\ Massachusetts Institute of Technology, \\ Cambridge, MA 02139, USA.

\section{Correspondence} \\ Tom Beucler, Department of Earth \\ Atmospheric and Planetary Sciences, \\ Massachusetts Institute of Technology, \\ Cambridge, MA 02139, USA. \\ Email: tom.beucler@gmail.com \\ Funding information \\ Tom Beucler: NSF AGS-1520683, Timothy \\ Cronin: NSF AGS-1623218
}

There is no consensus on the physical mechanisms controlling the scale at which convective activity organizes near the Equator. Here, we introduce a diagnostic framework relating the evolution of the length scale of convective aggregation to the net radiative heating, the surface enthalpy flux, and horizontal energy transport. We evaluate these expansion tendencies of convective aggregation in twenty high-resolution cloud-permitting simulations of radiativeconvective equilibrium. While both radiative fluxes contribute to convective aggregation, the net longwave radiative flux operates at large scales (1000-5000 km) and stretches the size of moist and dry regions, while the net shortwave flux operates at smaller scales $(500-2000 \mathrm{~km})$ and shrinks it. The surface flux expansion tendency is dominated by convective gustiness, which acts to aggregate convective activity at smaller scales (500-3000 km).

\section{KEYWORDS}

Convection, Turbulence, Self-aggregation, Moist static energy, Radiation, Surface flux, Spectral analysis.

\section{1 | INTRODUCTION}

7 To first order, the size of extra-tropical storms is set by the Rossby deformation radius, which scales inversely with the 8 Coriolis parameter. However, what sets the scale at which convective activity organizes near the Equator, where the 9 Coriolis parameter is small, remains an open question. Observations of monsoonal depressions (e.g. Figure 12 of Houze 10 [1989]) and the Madden-Julian oscillation (e.g. Figure 3b of Zhang 2005] indicate that convectively active regions near 
the Equator span scales from $100 \mathrm{~km}$ to $15,000 \mathrm{~km}$, but we lack a simple framework that would combine internal and external influences on the scale of these regions.

Fundamental understanding of convection has been bolstered by simulating radiative-convective equilibrium, an idealized state of the Tropical atmosphere in which radiative cooling balances convective heating in the absence of Jateral energy transport (e.g. review by Ramanathan and Coakley [1978]) using cloud-permitting models (e.g. Tompkins and Craig (1998) and general circulation models with parametrized convection (e.g. Held et al. [2007]). High-resolution cloud-permitting simulations of non-rotating convection show the clustering of convective activity despite homogeneous boundary forcing; this phenomenon has been referred to as convective self-aggregation (e.g. Held et al. [1993], Bretherton et al. [2005]). Studies to date with square domains in three-dimensional cloud-permitting models, without rotation, have found at most one cluster in the self-aggregated state, regardless of the domain size. With only a single cluster in aggregated square domains, the length scale of self aggregation in such simulations does not correJate with other parameters such as resolution (e.g. Muller and Held [2012]) or surface temperature (e.g. Wing and Emanuel (2014)), limiting our physical understanding of the length scale of convective aggregation. However, using the Regional Atmospheric Modeling System, Posselt et al.|2012] showed that non-rotating simulations which used elongated-channel geometry exhibited multiple aggregated areas. Wing and Cronin [2016] came to the same conclusion using the System for Atmospheric Modeling (SAM). Arnold and Randall [2015] and Coppin and Bony [2015] showed that aquaplanet simulations using the Goddard Earth observing System Atmospheric General Circulation Model and the IPSL-CM5A-LR General Circulation Model, respectively, exhibited multiple convective clusters when run with parametrized convection. The same is true for more realistic aquaplanet simulations that include planetary rotation and latitudinal sea surface temperature gradients (e.g. Bretherton and Khairoutdinov 2015). These recent findings suggest that the length scale $L$ of convective aggregation may be regulated by physical mechanisms, rather than artifacts of the model configuration, in some simulations - and thus within the reach of physical understanding.

Since resolving both moist turbulent convective activity (1-10 km scale) and the scale of moist and dry regions associated with large-scale overturning circulations (100-1000 km scale) is computationally expensive, identifying the physical mechanisms that set the length scale $L$ of moist and dry regions remains an active research area. In the context of an aquaplanet model with parameterized convection, Grabowski and Moncrieff [2004] have proposed that the length scale associated with moisture-convection feedback on intra-seasonal timescales is related to the large-scale subsidence drying. By taking the product of the typical horizontal wind speed $\left(\sim 5 \mathrm{~m} \mathrm{~s}^{-1}\right)$ and a subsidence drying timescale ( $\sim 10 \mathrm{~d}$ ), they obtain a value $L \sim 5000 \mathrm{~km}$. In the context of elongated-channel simulations, Wing and Cronin 2016 proposed that $L$ is the distance needed to re-moisten a subsiding air parcel in the boundary layer, which scales as the ratio of the boundary layer height $(\sim 1 \mathrm{~km})$ to the surface enthalpy exchange coefficient $\left(\sim 2 \times 10^{-3}\right)$, leading to typical values of $500 \mathrm{~km}$. However, this simple scaling does not capture the decrease of length scale observed when the radiation scheme is changed [see Figure 7 of Wing and Cronin 2016] from the radiative transfer module of the National Center for Atmospheric Research Community Atmosphere Model version 3 [CAM, Collins et al. 2006] to the Rapid Radiative Transfer Model [RRTM, lacono et al. [2008]. In the context of two-dimensional cloud-permitting simulations with SAM, Yang [2017] proposed that $L$ scales like the product of the boundary layer height $(\sim 1 \mathrm{~km})$, the square root of the boundary layer density variations due to humidity variations $\left(\sim \sqrt{10^{-3}}\right.$, referred to as the virtual effect), and a combined parameter that includes momentum damping in the boundary layer, stratification and radiative cooling at the top of the boundary layer $\left(5 \times 10^{-4}\right)$. Although this scaling yields the correct order of magnitude for $L(\sim 1500$ $\mathrm{km}$ ), the theory requires knowledge of density and radiative cooling variations at the top of the boundary layer. The underlying physical mechanism proposed by Yang 2017] has been refined by Arnold and Putman [2018] in the context of the Goddard Earth Observing System Atmospheric General Circulation Model, who suggested that density variations in the boundary layer due to temperature variations constrain $L$ to be less than $3000-4000 \mathrm{~km}$ through boundary layer 
momentum balance.

The previous theories predict the order of magnitude of $L$ and some of its dependencies (surface temperature, boundary layer properties, etc.) by assuming a dominant physical mechanism a priori. This paper takes an alternative approach by formulating a budget for $L$ and diagnosing the contributions to its evolution from different processes in three-dimensional cloud-permitting simulations with interactive radiation, surface fluxes and large-scale dynamics. We ask:

How do radiation, surface enthalpy fluxes and advection contribute to the emergence and evolution of a dominant length scale for convective aggregation?

In the spirit of the spectral analysis done in Arnold [2015] and in sections 5-7 of Bretherton and Khairoutdinov [2015, we develop a spectral diagnostic framework for the evolution of $L$ in section 2 and identify the controlling physical mechanisms from the results in section 3 .

\section{2 | METHOD}

Our goal is to quantify the roles of radiation, surface fluxes and advection in selecting the length scale of convective organization. First, we define a length scale $L$ for convective aggregation from the spatial spectrum of moist static energy (section 2.1. Then, we formulate a spectral budget that relates the evolution of $L$ to the vertically-integrated diabatic fluxes (section 2.2. We evaluate the radiative, surface flux and advective terms of this budget in a set of three-dimensional, cloud-permitting simulations across a range of sea surface temperatures, using SAM (section 2.3).

\section{1 | Definitions}

We use the column-integrated frozen moist static energy $H$ - interchangeably referred to as MSE in this paper - as a proxy for the organization of convection. $H$ has units $\mathrm{J} \mathrm{m}^{-2}$ and is defined as the sum of column internal energy, potential energy and latent heat:

$$
H \stackrel{\text { def }}{=} \int_{0}^{p_{s}} \frac{d p}{g}(\overbrace{c_{p} T}^{\text {Sensible heat }}+\underbrace{g z}_{\text {Potential energy }}+\overbrace{L_{v} q}^{\text {Lat. heat vap }}-\underbrace{L_{f} q_{i}}_{\text {Lat. heat fusion }}) \text {, }
$$

where $p_{s}$ is the surface pressure, $d p$ the differential pressure, $g$ the gravity constant, $c_{p}$ the specific heat capacity of dry air at constant pressure, $T$ the absolute temperature, $z$ the geopotential height, $L_{v}$ the latent heat of vaporization, $q$ the specific humidity, $L_{f}$ the latent heat of fusion and $q_{i}$ the condensed ice water mixing ratio. When convection is statistically homogeneous (a.k.a. "pop-corn" convection), then $H$ is also statistically homogeneous. However, when convection starts to organize, a large-scale overturning circulation develops (e.g. Muller and Bony (2015]). Regions of large-scale upwelling are moister (higher $H$ ) and have more active convection, while regions of large-scale downwelling are drier (lower $H$ ) and have less active convection. Therefore, a natural size for the organization of convection is the distance between regions of negative and positive spatial MSE anomalies. To gain a physical understanding of what sets this distance $L$, we formally define it as the spectral mean of the wavelength, weighted by the spatial power spectrum $\varphi$ of $H$ :

$$
L \stackrel{\text { def }}{=} \frac{\langle\lambda \varphi\rangle}{\langle\varphi\rangle},
$$


where the wavelength $\lambda$ is defined from the modulus $\|\boldsymbol{k}\|$ of the wave vector $\boldsymbol{k}$ in $n$ dimensions as:

$$
\lambda=\frac{2 \pi \sqrt{n}}{\|k\|} .
$$

The spatial spectrum of $H, \varphi$, is defined from the MSE spatial Fourier transform $\widehat{H}$ :

$$
\varphi \stackrel{\text { def }}{=} \widehat{H} \widehat{H}^{*},
$$

$$
\widehat{H} \stackrel{\text { def }}{=} \frac{1}{(2 \pi)^{\mathrm{n} / 2}} \int_{0}^{L_{\mathrm{dom}}} \exp (-\iota \boldsymbol{k} \cdot \boldsymbol{x}) H(\boldsymbol{x}) d \boldsymbol{x},
$$

where $\widehat{H}^{*}$ is the complex conjugate of $\widehat{H}, \boldsymbol{x}$ is the horizontal position vector in $n$ dimensions and $\iota$ is the unit imaginary number, $\iota^{2}=-1$ (note that we later use $i$ as an index to refer to distinct processes). In equation 5 , the domain is assumed to be rectangular and to span from the origin of the coordinate system $\boldsymbol{x}=\mathbf{0}$ to the opposite corner of the domain $\boldsymbol{x}=\boldsymbol{L}_{\mathrm{dom}}$. To improve the readability of equation 2 we have introduced the spectral average \langle\rangle :

$$
\langle X\rangle \stackrel{\text { def }}{=} \frac{\int_{k_{0}}^{k_{N}} X(k) d k}{\int_{k_{0}}^{k} d k},
$$

where $\boldsymbol{k}_{0}$ is the wavenumber with smallest non-zero magnitude and $\boldsymbol{k}_{\boldsymbol{N}}$ is the Nyquist wavenumber. In a rectangular three-dimensional model of horizontal domain size $\left(L_{x}, L_{y}\right)$ and resolution $(d x, d y)$, the integration bounds are $k_{0}=$ $2 \pi\left(L_{x}^{-1}, L_{y}^{-1}\right)$ and $k_{N}=\pi\left(d x^{-1}, d y^{-1}\right)$. In applying equation 3 to a three-dimensional domain with equal horizontal lengths $L_{x}=L_{y}$, the pre-factor $2 \pi \sqrt{2}$ ensures that $L$ equals the segment length $L_{x}$ if all the power $\varphi$ is concentrated in the largest wave vector $\boldsymbol{k}_{0}$. In this study, we have made a simple choice for the definition of $L$ given by equation 2 Alternatively, we could have used the integral scale: $2 \pi \sqrt{n}\langle\varphi\rangle /\langle\|\boldsymbol{k}\| \varphi\rangle$ to measure the scale of MSE spatial anomalies (e.g. equation 8.45 of Vallis [2006 and equations 7-8 of Wing and Cronin [2016]). It is possible to generalize the definition of $L$ to: $L \stackrel{\text { def }}{=} 2 \pi \sqrt{n}\left\langle\|\boldsymbol{k}\|^{p} \varphi\right\rangle^{1 / q}\left\langle\|\boldsymbol{k}\|^{p+q} \varphi\right\rangle^{-1 / q}$, where $(p, q) \in \mathbb{Z}^{2}$ are integers. Note that $(p, q)=(-1,1)$ in our definition of $L$ (equation 2). The choice of $(p, q)$ determines the relative weighting of the different parts of the spectrum $\varphi$. For example, if $(p+1)$ is larger than the spectral slope $s$ such that $\varphi \sim\|\boldsymbol{k}\|^{-s}$, then $L$ is determined by the small-scale contributions to the MSE spectrum, while $L$ is dominated by the large-scale contributions to the MSE spectrum if $(p+q+1)$ is smaller than $s$. Therefore, $L$ cannot describe the MSE spectrum in its full complexity, and we compare different choices for $L$ in appendix $\mathrm{A}$ Nevertheless, having a single metric $L(t)$ to quantify the spatial organization of MSE reduces the problem to a single time-dependent variable, and we proceed to relate its timeevolution to the diabatic fluxes of MSE.

\section{2 | Budgets}

\subsubsection{Frozen moist static energy budget}

The column frozen moist static energy $H$ is conserved under vertical convective mixing, and altered by the net moist static energy flux (in $\mathrm{Wm}^{-2}$ ) through the boundaries of the atmospheric column in consideration. The total flux is the sum of the net radiative flux at the top and bottom of the atmosphere $\dot{H}_{\text {rad }}$, the net surface enthalpy flux $\dot{H}_{\text {sf }}$, and the 
horizontal flux of moist static energy $\dot{H}_{\text {adv }}$ (referred to as the advective flux and defined as the mass-weighted column integral of the three-dimensional advection of local frozen moist static energy). The evolution equation of $H$ can be written:

$$
\frac{\partial H}{\partial t}=\dot{H}_{\mathrm{lw}}+\dot{H}_{\mathrm{sw}}+\dot{H}_{\mathrm{sf}}+\dot{H}_{\mathrm{adv}}
$$

where $t$ is time (in s). When averaged in space and time, the net longwave flux $\dot{H}_{\mathrm{Iw}}$ is negative as the atmosphere cools to space, the net shortwave flux $\dot{H}_{\text {sw }}$ positive as the atmosphere absorbs solar radiation, and the surface enthalpy flux $\dot{H}_{\text {sf }}$ positive as the surface enthalpy is greater than the enthalpy of the air above it. In RCE, these three tendencies balance out to zero in the absence of horizontal MSE fluxes $\dot{H}_{\mathrm{adv}}$. As an atmospheric circulation develops, the MSE advective flux $\dot{H}_{\text {adv }}$ becomes positive if there is a net MSE import into the column, and negative in the case of a net MSE export. Equation7holds for all length scales, which means that at a given scale, the value of each flux increases or decreases the MSE at that scale. This statement can be made mathematically explicit by working in spectral space.

\subsubsection{Budget for the spatial spectrum of frozen moist static energy}

To derive the budget for the spatial spectrum of frozen moist static energy $\varphi$, we start by taking the time-derivative of its definition (equation 4):

$$
\frac{\partial \varphi}{\partial t} \stackrel{\operatorname{def}}{=} \frac{\partial\left(\widehat{H}^{*} \widehat{H}\right)}{\partial t}=2 \operatorname{Re}\left(\widehat{H}^{*} \frac{\partial \widehat{H}}{\partial t}\right)
$$

where Re refers to the real part of a complex number. We then take the spatial Fourier transform of the frozen moist static energy's budget (equation 7 :

$$
\frac{\partial \widehat{H}}{\partial t}=\frac{\widehat{\partial H}}{\partial t}=\widehat{\dot{H}_{\mathrm{Iw}}}+\widehat{\dot{H}_{\mathrm{sw}}}+\widehat{\dot{H}}_{\mathrm{sf}}+\widehat{\dot{H}_{\mathrm{adv}}}
$$

Substituting equation 9 into equation 8 yields:

$$
\frac{\partial \varphi}{\partial t}=\dot{\varphi}_{\mathrm{lw}}+\dot{\varphi}_{\mathrm{sw}}+\dot{\varphi}_{\mathrm{sf}}+\dot{\varphi}_{\mathrm{adv}}
$$

where each spectral tendency $\dot{\varphi}_{i}$ is related to the corresponding flux $\dot{H}_{i}$ via:

$$
\forall i, \quad \dot{\varphi}_{i}=2 \operatorname{Re}\left\{\widehat{H}^{*} \widehat{\dot{H}}_{i}\right\}
$$

where $\forall i$ indicates that equation 11 is valid for each individual process $\dot{H}_{i}$. Note that we use $i$ hereafter as a general subscript to refer to any of the processes that modify $H$ : longwave radiation, shortwave radiation, surface fluxes, or advection. Equation 10 relates the evolution of the spectrum $\varphi$ to the spectral tendencies $\dot{\varphi}_{i}$ for each wave vector $\boldsymbol{k}$. 


\subsection{3 | Convective aggregation length scale budget}

To derive the budget for the convective aggregation length scale $L$, we start by taking the logarithmic time-derivative of its definition (equation 2):

$$
\frac{1}{L} \frac{\partial L}{\partial t} \stackrel{\text { def }}{=} \frac{1}{\langle\lambda \varphi\rangle}\left\langle\lambda \frac{\partial \varphi}{\partial t}\right\rangle-\frac{1}{\langle\varphi\rangle}\left\langle\frac{\partial \varphi}{\partial t}\right\rangle
$$

We then substitute the right-hand side of the $\varphi$-evolution equation 10 into equation 12 . This allows us to write the right-hand side of equation 12 as a sum of individual expansion tendencies (in units $\mathrm{m} \mathrm{s}^{-1}$ ), which we refer to as the budget for the convective aggregation length scale $L$ :

$$
\frac{\partial L}{\partial t}=\dot{L}_{\mathrm{iw}}+\dot{L}_{\mathrm{sw}}+\dot{L_{\mathrm{sf}}}+\dot{L}_{\mathrm{adv}}
$$

Note that we use 'rate' to describe the relative growth or decay (in inverse time units) of the total MSE spatial variance $\langle\varphi\rangle$ (aggregation rate) or the convective aggregation length scale $L$ (expansion rate), while 'tendency' refers to the time-derivative of a variable. According to equation 12 each expansion rate is given by:

$$
\begin{aligned}
\forall i, \frac{\dot{L}_{i}}{L} & =\frac{\left\langle\lambda \dot{\varphi}_{i}\right\rangle}{\langle\lambda \varphi\rangle}-\frac{\left\langle\dot{\varphi}_{i}\right\rangle}{\langle\varphi\rangle} \\
& =\frac{\left\langle\dot{\varphi}_{i}\right\rangle}{\langle\varphi\rangle}\left(\frac{\left\langle\lambda \dot{\varphi}_{i}\right\rangle}{\left\langle\dot{\varphi}_{i}\right\rangle} \frac{\langle\varphi\rangle}{\langle\lambda \varphi\rangle}-1\right)
\end{aligned}
$$

The tendencies of $L$, given by equation 14 can be re-written as:

$$
\forall i, \dot{L}_{i}=\underbrace{\frac{\left\langle\dot{\varphi}_{i}\right\rangle}{\langle\varphi\rangle}}_{\text {Aggregation rate }} \overbrace{\left(\mathcal{L}_{i}-L\right)}^{\text {Length scale factor }},
$$

$$
\mathcal{L}_{i} \stackrel{\text { def }}{=} \frac{\left\langle\lambda \dot{\varphi}_{i}\right\rangle}{\left\langle\dot{\varphi}_{i}\right\rangle}
$$

where $\mathcal{L}_{i}$ is the length scale associated with the spectral tendency $\dot{\varphi}_{i}$, analogous to the convective aggregation length scale $L$ (equation 2], but based on the tendency of the spectrum of $H$ due to a particular physical process, rather than on the spectrum of $H$ itself. To build intuition about what sets $\mathcal{L}_{i}$, we consider three examples:

1. A diabatic process that linearly damps spatial MSE anomalies $H^{\prime}$ at a rate $\alpha$, such as the enthalpy disequilibrium component of the surface flux: $\dot{H}_{\text {dis }} \approx-\alpha H^{\prime}$. Following equation 11 its spectral tendency linearly damps the MSE spectrum for non-zero wave vectors: $\dot{\varphi}_{\text {dis }}=2 \operatorname{Re}\left\{\widehat{H}^{*} \widehat{(-\alpha H)}\right\}=-2 \alpha \varphi$. Finally, equation 16 yields: $\mathcal{L}_{\text {dis }}=$ $\langle-2 \alpha \varphi \lambda\rangle /\langle-2 \alpha \varphi\rangle=L$, showing that a scale-neutral diabatic process that acts to linearly damp or amplify the MSE anomaly has the same scale $L$ as the MSE anomaly itself.

2. A scale-selective process that preferentially amplifies MSE anomalies at large scales, such as the net longwave flux convergence: $\widehat{\dot{H}_{\mathrm{lw}}}=\alpha\left(\lambda / \lambda_{0}\right)^{\mu} \widehat{H^{\prime}}$, where $\alpha, \mu$ and $\lambda_{0}$ are constant positive rate, longwave spectral exponent and characteristic wavenumber, respectively. Following equation 11 its spectral tendency is strongest at large scales: $\dot{\varphi}_{\text {Iw }}=2 \operatorname{Re}\left\{\widehat{H}^{*} \alpha \widehat{\left(\lambda / \lambda_{0}\right)^{\mu}} H\right\}=2 \alpha\left(\lambda / \lambda_{0}\right)^{\mu} \varphi$. At this point, it is useful to note that except for small wave vectors, the 
MSE spectrum quickly decays with the wave vector amplitude: $\varphi=\varphi_{0} \lambda^{s}$, where $\varphi_{0}$ is a constant spectral amplitude and we require the spectral slope $s$ be strictly larger than 2 . Typically, $s \approx 3$ above wavenumber $(2 \pi) L^{-1}$, as can be seen in panel 6b of Wing and Cronin [2016]; if the spectral slope $s$ was smaller than 2, our definition of the convective aggregation length scale $L$ would depend on the model's horizontal grid spacing through the Nyquist wave vector $\boldsymbol{k}_{\boldsymbol{N}}$. The diabatic length scale for that scale-selective process is calculated using equation 16

$$
\frac{\mathcal{L}_{\mathrm{lw}}}{L}=\frac{\left\langle\lambda \dot{\varphi}_{\mid \mathrm{w}}\right\rangle}{\left\langle\dot{\varphi}_{\mathrm{Iw}}\right\rangle} \frac{\langle\varphi\rangle}{\langle\lambda \varphi\rangle}=\frac{\left\langle\lambda^{\mu+s+1}\right\rangle}{\left\langle\lambda^{\mu+s}\right\rangle} \frac{\left\langle\lambda^{s}\right\rangle}{\left\langle\lambda^{s+1}\right\rangle} \approx \underbrace{1+\overbrace{\frac{\mu}{(\mu+s)(s-1)}}^{>0}}_{>1} .
$$

Therefore, a process $i$ that amplifies MSE anomalies at large scales has an associated length scale $\mathcal{L}_{i}$ larger than the MSE anomaly scale $L$, by a factor that depends on the MSE spectral slope $s$ and the spectral exponent of the process, $\mu$.

3. A scale-selective process that preferentially amplifies MSE anomalies at small scales, such as the net shortwave flux convergence. By applying the previous model and equation 17 to the case of a constant negative spectral exponent $\mu \in]-s, 0[$, the length scale $\mathcal{L}$ associated with that process is smaller than the MSE anomaly scale $L$.

Equation 15 allows us to interpret each tendency $\dot{L}_{i}$ as a product of two factors. In appendix A.2 we prove that the factor $\left\langle\dot{\varphi}_{i}\right\rangle /\langle\varphi\rangle$ is equal to the growth rate of moist static energy spatial variance in inverse time units (e.g. the terms on the right-hand side of equation 4 of Wing and Cronin |2016]; see sections 3.1-3.4 of Wing et al.|2017] for a review of their physical interpretation). The second factor is a length scale factor that vanishes if the convective aggregation length scale $L$ equals the length scale $\mathcal{L}_{i}$. We focus on the physical interpretation of equation 15 in section 2.5

\section{3 | Simulation design}

We use a series of twenty simulations to understand the dependence of the radiative, surface flux and advective expansion tendencies on the surface temperature, radiation scheme and domain geometry. Following Wing and Cronin [2016], radiative-convective equilibrium (RCE) is simulated using version 6.8.2 of SAM (e.g. Khairoutdinov and Randall [2003]); we contrast the characteristics of the cloud-permitting experiments in table 1 Seventeen of the twenty simulations were provided by Allison Wing; the reader interested in the setup and general characteristics of the longchannel simulations is referred to sections 3 and 4 of Wing and Cronin [2016], respectively, while the characteristics of the square simulation are described in sections 7.2 and 7.5 of Wing and Cronin [2016. The big square-domain simulation uses the same set-up as the square-domain simulations (Allison Wing, personal communication). We also run 3 mechanism denial experiments (MD), in which the radiative heating, the surface enthalpy fluxes, and both the radiative heating and the surface enthalpy fluxes, are horizontally homogenized at each vertical level and at the beginning of each timestep. The evolution of the moist static energy field $H$, averaged over the short dimension of the channel for these 3 experiments, is depicted in Figure 1 as well as the evolution of $L$. The evolution of the moist static energy field $H$ of the three square experiments is depicted in Figure 2 along with the corresponding MSE anomaly scale $L$. 


\begin{tabular}{|c|c|c|c|c|}
\hline Acronym & LC & MD & SQ & BSQ \\
\hline \# simulations & 14 & 3 & 2 & 1 \\
\hline$T_{s}(\mathrm{~K})$ & $280 / 285 / . . / 310$ & 300 & 305 & 305 \\
\hline Dom. size $(\mathrm{km})$ & $12288 \times 192$ & $12288 \times 192$ & $1536 \times 1536$ & $3072 \times 3072$ \\
\hline Radiation & CAM/RRTM & CAM & CAM/RRTM & RRTM \\
\hline
\end{tabular}

TAB LE 1 Characteristics of the twenty cloud-permitting experiments used in this paper. From top to bottom: Acronym given to the series of simulations (LC: Long-channel domain, MD: Mechanism-denial experiments, SQ: Square domain, BSQ: Big square domain), number of simulations in each series, fixed surface temperature $T_{s}$, domain size and radiation scheme. 


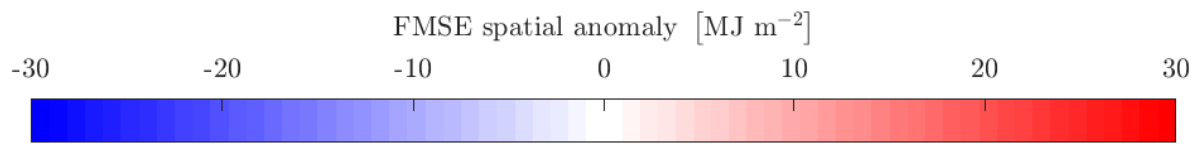

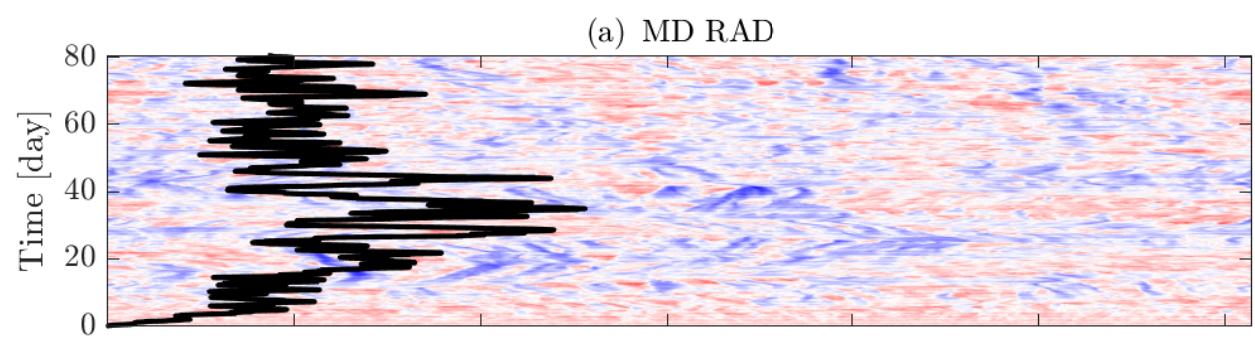

(b) MD SFC

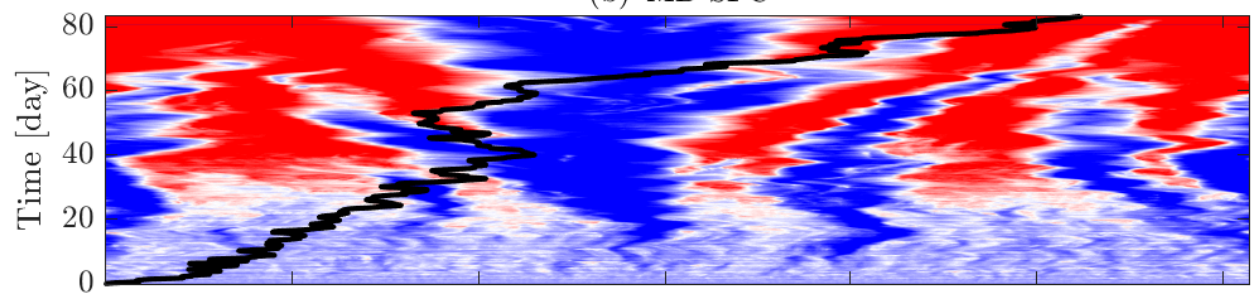

(c) $\mathrm{MD} \mathrm{RAD}+\mathrm{SFC}$

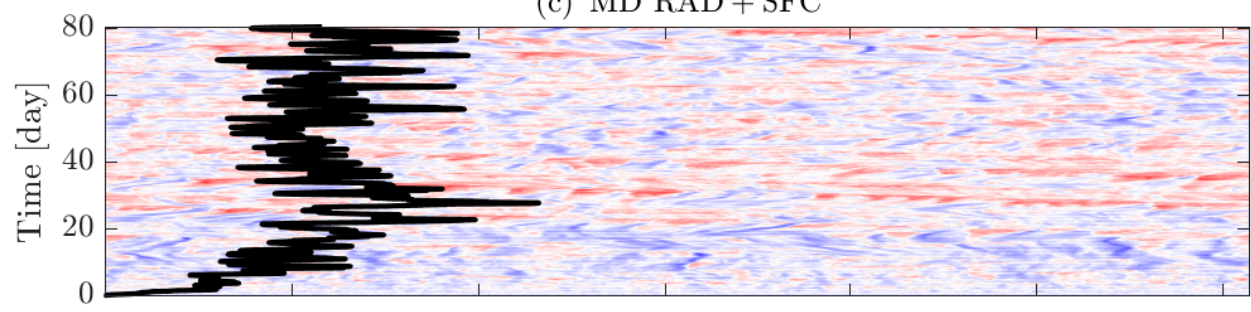

(d) LC300 CAM

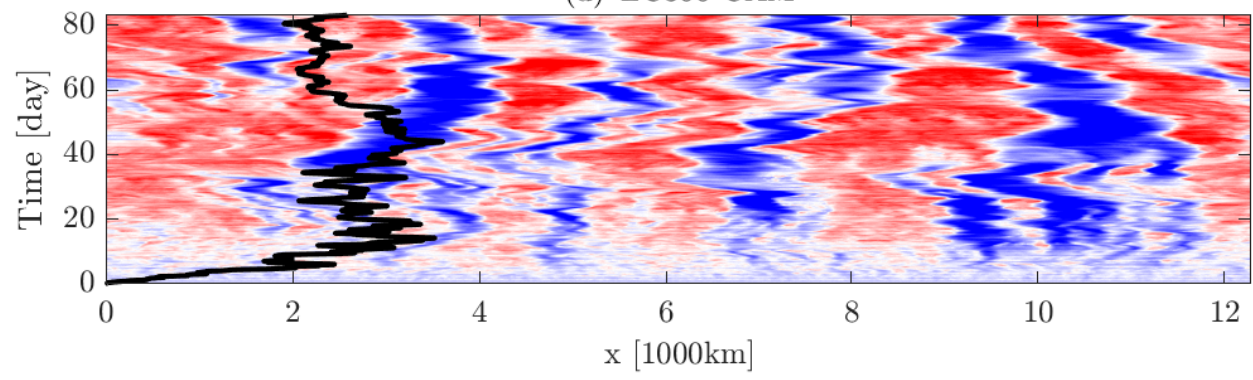

FIGURE 1 Hovmöller diagram of the frozen moist static energy spatial anomaly $H^{\prime}$ (in $\mathrm{MJ} \mathrm{m}^{-2}$ ) for the three MD experiments and the control LC300 CAM experiment. The vertical axis is time (in days) and the horizontal axis is $x$ (in $1000 \mathrm{~km}$ ). The anomaly has been spatially-averaged in the $y$-direction. The distance between the left vertical axis and the thick, black line represents the one-dimensional time-varying convective aggregation length scale $L(t)$ (in $1000 \mathrm{~km}$, defined in equation 2. 

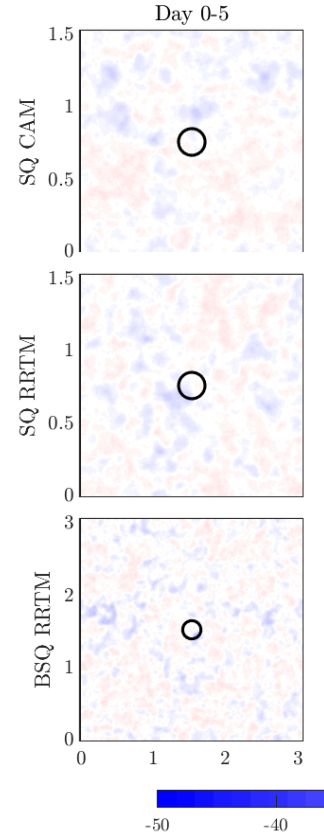

40
Day 20-25
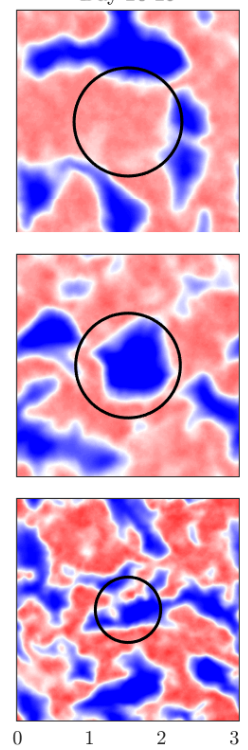

Day 40-45
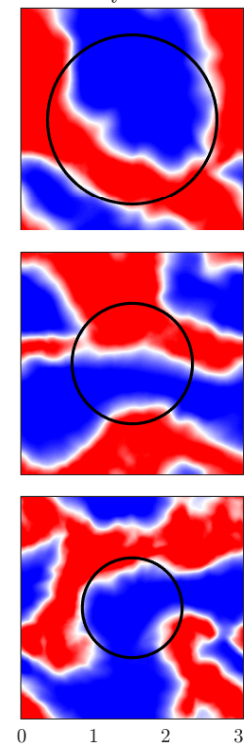

Day 60-65
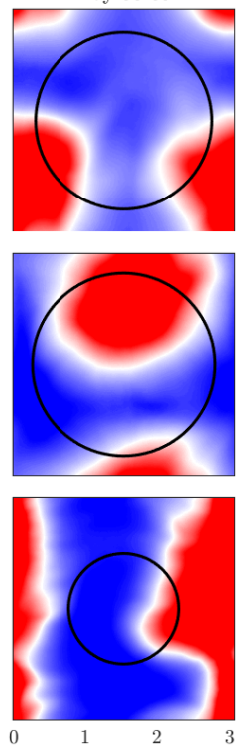

Day 80-85
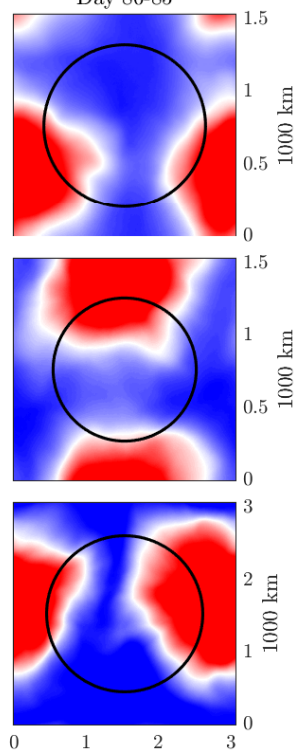
머용

F IG URE 2 Snapshots of the frozen moist static energy spatial anomaly $H^{\prime}$, averaged in time over periods of five days (in $\mathrm{MJ} \mathrm{m}^{-2}$ ) for the two SQ experiments and the BSQ experiment. For each snapshot of the doubly-periodic square domain, the horizontal axis is $x$ (in 1000km) and the vertical axis is $y$ (in $1000 \mathrm{~km}$ ). The radius of each black circle represents the two-dimensional time-varying convective aggregation length scale $L(t)$ (in 1000km, defined in equation 2.

\subsection{Calculation of the budget terms}

Hourly-averages of $H$ and the diabatic fluxes $\dot{H}_{\mathrm{lw}}, \dot{H}_{\mathrm{sw}}, \dot{H}_{\mathrm{sf}}$ are computed every hour, using their values at every time step. We call the radiation module every six minutes, calculate hypothetical radiative heating profiles without clouds for diagnostic purposes, and obtain longwave and shortwave "clear-sky" radiative fluxes: $\dot{H}_{\text {Iwcs }}, \dot{H}_{\text {swcs. }}$ The advective flux, $\dot{H}_{\text {adv }}$, is computed as a residual from equation 7. We verify that $\dot{H}_{\text {adv }}$ calculated as a residual has the same behavior as $\dot{H}_{\text {adv }}$ computed from the three-dimensional fields of $H$ and velocity (for the standard experiment LC300 with CAM radiation, the domain-averaged aggregation feedback $\left\langle\dot{\varphi}_{\text {adv }}\right\rangle$ matches within $20 \%$ if we use three-dimensional snapshots in time of $H$ and velocity to compute $\dot{H}_{\text {adv }}$ directly). We calculate the Fourier transform of $H$ and the MSE tendencies $\dot{H}_{i}$ using the MATLAB Fast Fourier Transform, which uses the C library FFTW (e.g. Frigo and Johnson 2005]). For the LC and MD simulations, the spatial variability in the long direction is more than 10 times the spatial variability in the short direction once convection has aggregated. Therefore, we average $H$ and $\dot{H}_{i}$ in the short direction before computing one-dimensional Fourier transforms in the long direction to calculate the moist static energy spectrum $\varphi$ and the spectral tendencies $\dot{\varphi}_{i}$. The spectral tendencies $\dot{\varphi}_{i}$ would have more power at smaller wavelengths $\lambda$ if the Fourier transform in the long direction was applied before averaging in the short direction, which would be less representative of the $y$-averaged spatio-temporal variability depicted in Figure 1 . For the SQ and BSQ simulations, we compute two-dimensional Fourier transforms of $H$ and $\dot{H}_{i}$ in the horizontal to obtain $\varphi$ and $\dot{\varphi}_{i}$. In all cases, following 
equation 6 we integrate $\varphi$ and $\dot{\varphi}_{i}$ between the smallest non-negative wavenumber and the Nyquist wavenumber to compute $L$ and $L_{i}$.

\section{5 | Interpretation of the expansion tendency}

For a given process (e.g., net longwave heating $\dot{H}_{\mathrm{IW}}$ ), equation 15 relates:

1. The longwave expansion tendency $L_{\mathrm{iw}}$, in units $\mathrm{m} \mathrm{s}^{-1}$. This tendency can be interpreted as the velocity at which longwave radiation stretches (if $L_{\mathrm{iw}}>0$ ) or shrinks (if $L_{\mathrm{iw}}<0$ ) the size of moist and dry regions (see equation 13 .

2. The longwave aggregation rate $\left\langle\dot{\varphi}_{\mid \mathrm{w}}\right\rangle /\langle\varphi\rangle$, in units day ${ }^{-1}$, which is the longwave contribution to the rate of increase in MSE spatial variance (a standard metric for the rate at which convection aggregates, e.g. section 3 of Wing et al. [2017]). If $\left\langle\dot{\varphi}_{\mid \mathrm{w}}\right\rangle>0$, the moist static energy spatial variance is reinforced and convection aggregates. If $\left\langle\dot{\varphi}_{\mid \mathrm{w}}\right\rangle<0$, the moist static energy field is smoothed and convection disaggregates.

3. The convective aggregation length scale $L$, which measures the typical size of moist and dry regions (see equation 2.

4. The longwave length scale $\mathcal{L}_{\mathrm{lw}}$, which measures the scale at which the longwave heating acts to aggregate convection.

If the aggregation tendency is positive $\left(\left\langle\dot{\varphi}_{\mid \mathrm{w}}\right\rangle>0\right)$ and acts at a larger scale than the convective aggregation length scale $\left(\mathcal{L}_{\text {Iw }}>L\right)$, then $L$ will exponentially relaxes towards $\mathcal{L}_{\text {Iw }}$, corresponding to a stretching $\left(L_{\text {Iw }}>0\right)$. We illustrate this case in Figure 3 and summarize the interpretation of equation 15 for all sign cases in Table 2

\begin{tabular}{|c|c|c|}
\hline & Small-scale: $\mathcal{L}_{i}<L$ & Large-scale: $\mathcal{L}_{i}>L$ \\
\hline \hline Aggregation: $\left\langle\dot{\varphi}_{i}\right\rangle>0$ & Shrinking: $\dot{L}_{i}<0$ & Stretching: $\dot{L}_{i}>0$ \\
\hline Disaggregation: $\left\langle\dot{\varphi}_{i}\right\rangle<0$ & Stretching: $\dot{L}_{i}>0$ & Shrinking: $\dot{L}_{i}<0$ \\
\hline
\end{tabular}

TAB LE 2 Guide for interpreting equation 15 which relates the expansion tendencies $\dot{L}_{i}$ to the aggregation rates $\left\langle\dot{\varphi}_{i}\right\rangle /\langle\varphi\rangle$ and the diabatic scales $\mathcal{L}_{i}$.

Aggregation at smaller scales $\mathcal{L}_{i}<L$ will make $L$ exponentially relax towards $\mathcal{L}_{i}$, corresponding to a shrinking (top-left case: $\dot{L}_{i}<0$ ). When the aggregation rate is negative (second row), $\mathcal{L}_{i}$ represents the scale at which MSE power is most strongly damped and acts as a repeller for the scale $L$ of MSE anomalies: repelling from small-scales leads to a stretching (bottom-left case: $\dot{L}_{i}>0$ ) while repelling from large-scales leads to a shrinking (bottom-right case: $\dot{L}_{i}<0$ ). Finally, when a process $\dot{H}_{i}$ acts at a scale $\mathcal{L}_{i}$ that is equal to the convective aggregation scale $L$, then it does not affect the scale of convective activity and $\dot{L}_{i}=0$. 


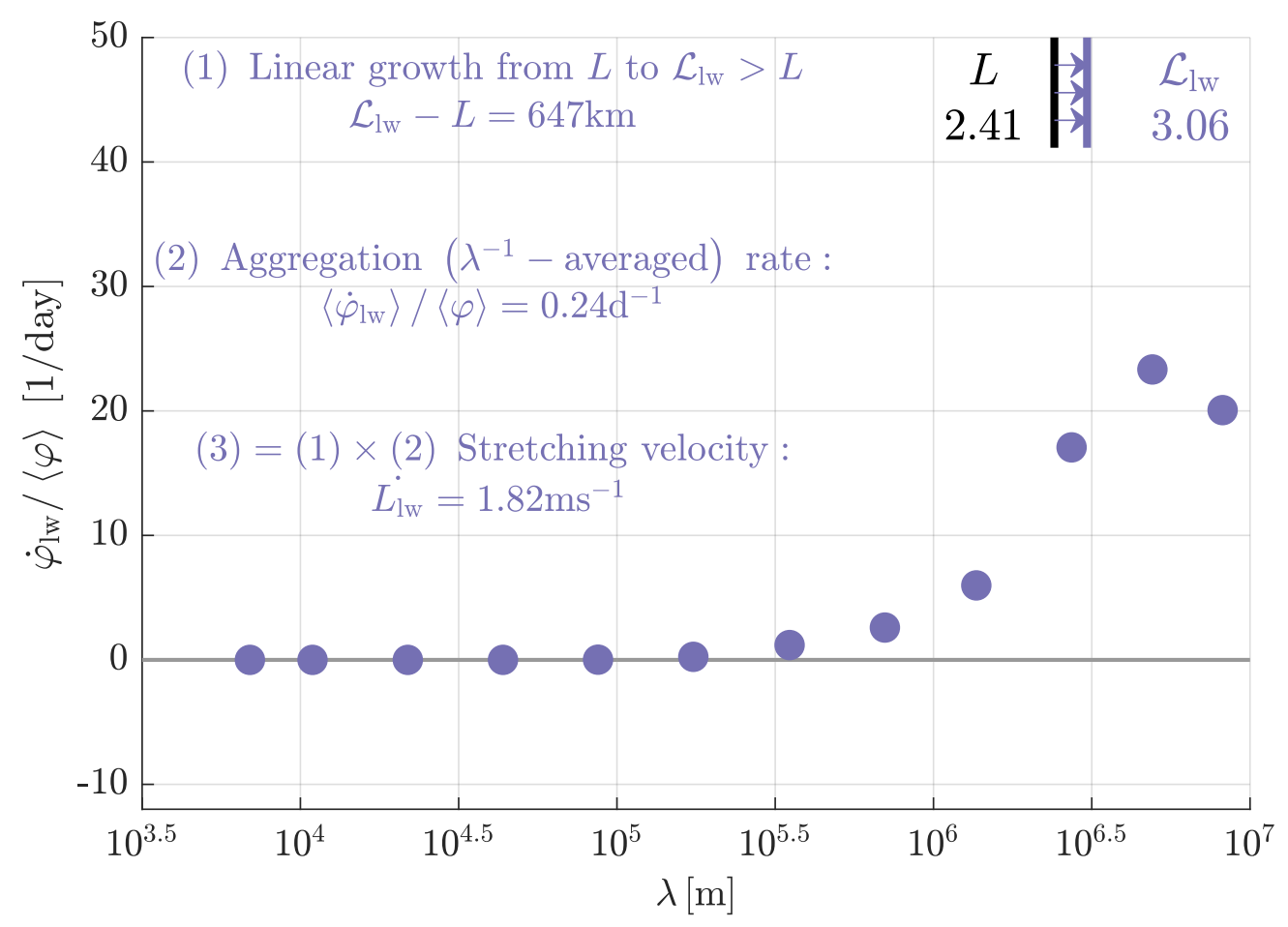

FIGURE 3 We use the time-averaged data from the first 30 days of the LC 300 simulation as an example. (Black line) Time-averaged convective aggregation length scale $L$ (in day ${ }^{-1}$ ).

(Blue line) Length scale $\mathcal{L}_{\text {Iw }}$ of the longwave tendency $\dot{\varphi}_{\text {Iw }}$.

(1-Arrows) Longwave radiation tries to "stretch" the convective aggregation length scale $L$ to its preferred length scale $\mathcal{L}_{\mid \mathrm{w}}$, because $\left\langle\dot{\varphi}_{\mid \mathrm{w}}\right\rangle>0$ and $\mathcal{L}_{\mathrm{lw}}>L$.

(2-Dots) Normalized longwave spectral rates $\dot{\varphi}_{\mid \mathrm{w}} /\langle\varphi\rangle$ (in day $^{-1}$ ) for each wavelength $\lambda$ (in $1000 \mathrm{~km}$ ). The spectral-average of the spectral rates equals the longwave aggregation rate $\left\langle\dot{\varphi}_{\mid \mathrm{w}}\right\rangle /\langle\varphi\rangle$ (in day $^{-1}$ ).

(3-expansion tendency) The combination of the positive longwave aggregation rate $\left\langle\dot{\varphi}_{\mid \mathrm{w}}\right\rangle /\langle\varphi\rangle>0$ with the growth from $L$ to $\mathcal{L}_{\text {Iw }}>L$ leads to a positive longwave expansion tendency $L_{\text {iw }}>0$.

\section{3 | RESULTS}

We show the evolution of the convective aggregation length scale $L$ in section 3.1 and the evolution of the tendencies $\dot{L}_{i}$ and $\dot{\varphi}_{i}$ in section 3.2 . We then investigate the physics of the radiative (section 3.3. and of the surface flux (section 3.4. tendencies.

\subsection{Convective aggregation length scales}

All experiments are initiated with a horizontally homogeneous state and small thermal noise $(\sim 0.05 \mathrm{~K}$ at the surface, decaying to zero at an elevation of 500 meters), and therefore start with very small MSE anomalies (less than $0.1 \mathrm{MJ} \mathrm{m}^{-2}$ ). A horizontally homogeneous state implies small $L$ (left of Figure44 ) and small degree of aggregation (left of Figure 4p). 
As convection organizes, the MSE power spectrum is:

- Enriched overall, which increases the spectrally-averaged MSE power $\langle\varphi\rangle$, or equivalently the spatial MSE anomalies (first month of Figure $4 \mathrm{p}$ ).

- Preferentially enriched at long wavelengths, which increases the MSE anomaly scale $L$ (first month of Figure 4 a).

In physical space, MSE anomalies amplify, which is referred to as convective self-aggregation. In the long-channel experiments (e.g. Figure 1 d), aggregated bands appear on the Hovmoller diagram, while aggregated moist and dry "blobs" appear in the square-geometry experiments (Figure 2). The convective aggregation length scale $L$ (marked with black lines in both figures) reasonably follows the average MSE anomaly size, and a discussion on the advantages of different definitions of $L$ can be found in appendix A Based on the degree of aggregation, it is conceptually useful to distinguish two phases:

- A "growth phase", in which convection aggregates and MSE spatial anomalies increase. To simplify the comparison among different experiments, we define this phase as the first month of the simulation (until the end of the 30th day). In the reference simulation LC300CAM, the weekly-averaged MSE spatial standard deviation at the end of the first month is $14.8 \mathrm{MJ} \mathrm{m}^{-2}, 84 \%$ of the time-averaged MSE standard deviation after the first month of simulation $\left(17.6 \mathrm{MJ} \mathrm{m}^{-2}\right)$.

- A "mature phase", in which convection has reached a statistically steady degree of aggregation, and MSE spatial anomalies have relatively small oscillations compared to their large time-averaged value (indicated with parenthesis in Figure 4 .

We will use this breakdown into two temporal phases when presenting time-averaged data in the rest of the manuscript. The length scale $L$, however, does not closely follow this same evolution:

- During the first week or two, $L$ grows rapidly as high and low-MSE regions appear.

- After that, $L$ has large-amplitude oscillations as MSE power is exchanged between the longest wavelengths. Because our definition 2 gives the largest weight to the longest wavelength, $L$ is very sensitive to MSE power exchange between the two longest wavelengths (domain size and half-domain size). Consequently, cold simulations (LC280CAM, LC280RRTM, LC285CAM) and the MD SFC experiment, which have a significant amount of MSE power in the longest wavelength, can undergo very large changes despite having reached a statistically steady degree of aggregation.

In the "mature phase", the LC experiments which have colder surface temperatures and use the CAM radiation scheme generally have larger $L$, but the largest values of $L$ are seen in the experiment where surface fluxes are horizontally homogenized (MD SFC, where $L$ is still growing after 80 days of simulation). By contrast, the experiments in which radiative heating is made horizontally homogeneous (MD RAD and MD RADSFC, see purple lines in Figure 4 b), exhibit very weak aggregation given their surface temperature of 300K, with MSE spatial anomalies three to four times smaller than the control LC300CAM case. When $L$ is defined following equation 2 MD RAD and MD RADSFC exhibit $L$ values that are close to the control LC300CAM case because their power spectra $\varphi$ increases with $\lambda$ at large $\lambda$ (see Figure 43). When $L$ is defined as the integral scale (equation 21, in Appendix 4], it captures a flatter part of the weakly-aggregated power spectrum $\varphi$, and MD RAD and MD RAD SFC exhibit very small $L$ compared to the control experiment LC300CAM (see accompanying MATLAB code for Figure 4]. Finally, the square domain experiments (SQ) 
have the smallest $L$, despite being strongly aggregated given their surface temperatures of 305K. For these experiments, $L$ may still be limited by the domain size, which is suggested by the fact that the BSQ experiment's MSE anomaly scale $L$ (dotted black line in Figure 4 a) is almost twice as large as its SQ counterpart (dotted gray line in Figure 43). Furthermore, $L$ equals half the domain size in both cases during the mature phase, consistent with the cluster occupying half of the domain in Figure 2 The spatial organization of convection in the long-channel setting is relatively insensitive to the domain size as long as the domain's aspect ratio remains large, which is discussed in section 7.5 of Wing and Cronin [2016. To understand what causes $L$ to vary so much, we now study the evolution of its diabatic tendencies $\dot{L}_{i}$.

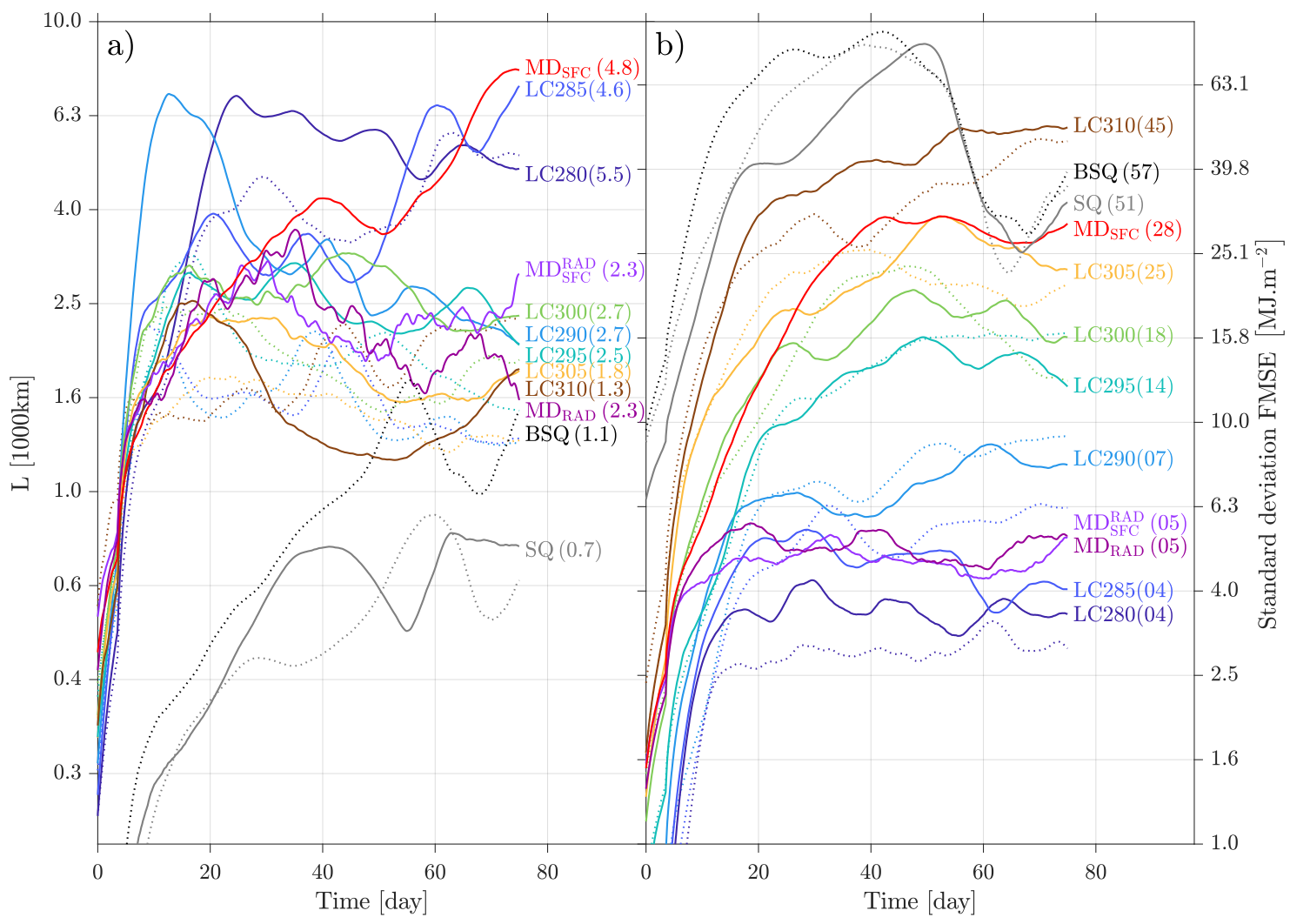

FIGURE 4 Evolution of (a) the convective aggregation length scale $L$ (in $1000 \mathrm{~km}$, defined in equation 2 and (b) the spatial standard deviation of MSE (in MJ. $\mathrm{m}^{-2}$ ) during the 75 days of each experiment. The solid lines represent the experiments using the CAM radiation schemes, and are identified using the acronyms defined in table 1 and the value of the fixed surface temperature. The dotted lines represent the experiments using the RRTM radiation scheme. We indicate the time-averaged value in the mature phase using parentheses, and use a time-moving-average of 1 week before plotting each variable. 


\subsection{Evolution of the length scale diabatic tendencies}

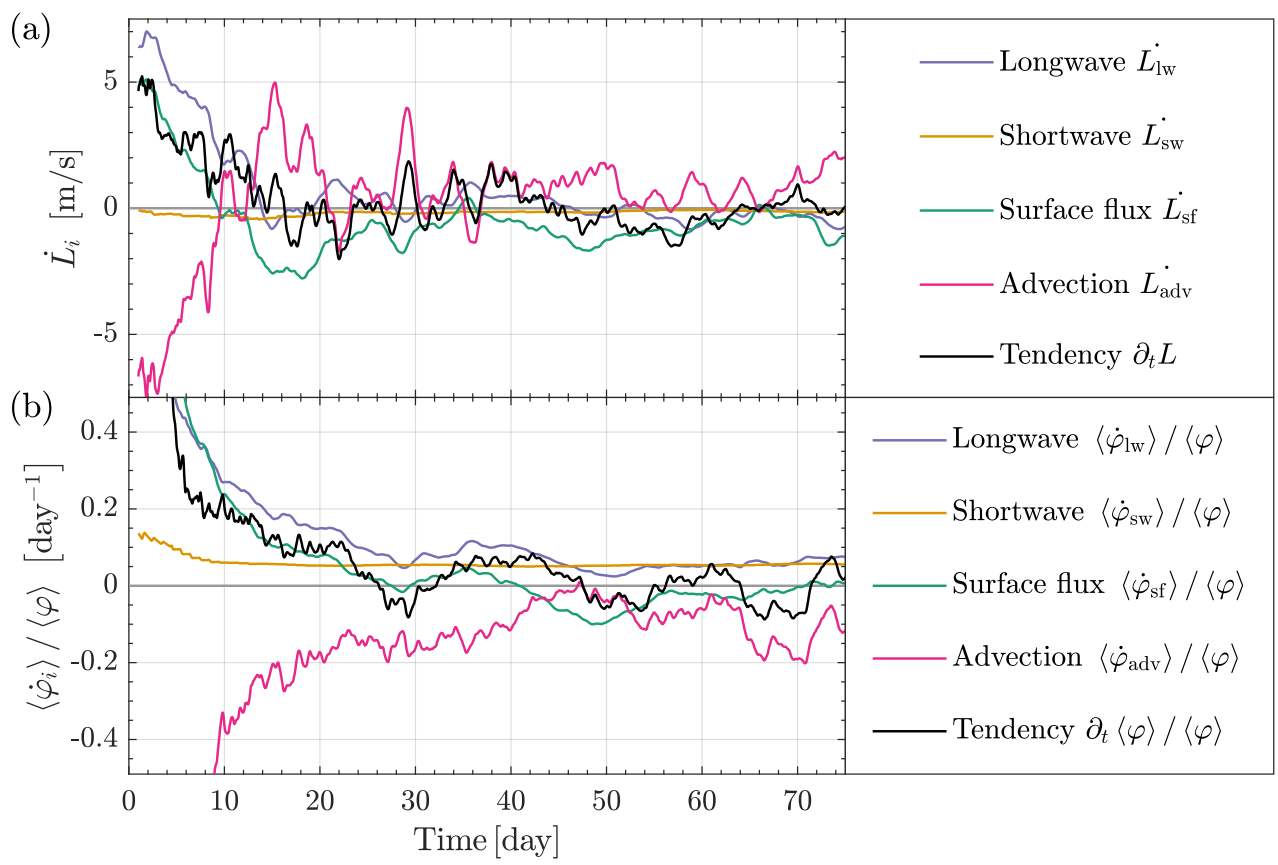

FIGURE 5 (a) Evolution of the expansion tendency $\dot{L}_{i}$ (in $\mathrm{ms}^{-1}$, on the right-hand side of equation 13 during the 75 days of experiment LC300 CAM. (b) Evolution of the aggregation rate $\left\langle\dot{\varphi}_{i}\right\rangle /\langle\varphi\rangle\left(\right.$ in day $^{-1}$ ) in LC300 CAM. Lines in both subplots are smoothed using a time-moving-average of 1 week.

We start by focusing on the LC300 CAM experiment, for which the evolution of $L(t)$ is depicted using a green line in Figure 4 The total forcing $L_{\text {tot }}$ (in $\mathrm{m} \mathrm{s}^{-1}$ ), represented with a black line in Figure 5 , is equal to the tendency of $L$ in time. Therefore, it is positive when $L$ grows ( $t<14.3$ day), and oscillates around zero afterwards ( $t>14.3$ day). Because the variability of $L$ is large in the mature phase, the amplitude of these oscillations sometimes exceeds that of $L_{\text {tot }}$ during the growth phase, challenging the idea of a uniquely-defined stationary size for convectively active regions during the mature phase. Following equation $13, L_{\text {tot }}$ can be decomposed into four expansion tendencies. The longwave tendency $L_{\text {iw }}$ (blue line) stretches $L$ at an average velocity of $1.8 \mathrm{~m} \mathrm{~s}^{-1}$ during the first month and has an overall neutral effect afterwards as $L$ oscillates around the longwave length scale $\mathcal{L}_{\mathrm{lw}}$. By contrast, the shortwave tendency $\dot{L}_{\text {sw }}$ (orange line) shrinks $L$ at an average velocity of $0.25 \mathrm{~m} \mathrm{~s}^{-1}$ during the first month and $0.13 \mathrm{~m} \mathrm{~s}^{-1}$ afterwards. The surface flux tendency $L_{\text {sf }}$ stretches $L$ for the first 12 days of the simulation before shrinking $L$ for the rest of the simulation. Finally, the advective tendency $L_{\text {adv }}$ exhibits the opposite behavior to $\dot{L}_{\text {sf }}$ in this particular case. During the growth phase, $L_{\text {adv }}$ 
shrinks $L$ at an average velocity of $0.54 \mathrm{~m} \mathrm{~s}^{-1}$ because it homogenizes MSE more at large scales than at small scales (bottom-right case of Table 2 consistent with the spectral Hövmoller plots of Arnold 2015), while $L_{\text {adv }}$ stretches $L$ at an average velocity of $0.93 \mathrm{~s}^{-1}$ during the mature phase because it homogenizes MSE more at small scales than at large scales (bottom-left case of Table 2]: The reader interested in the scale-by-scale MSE spectral tendencies $\dot{\varphi}_{\text {adv }}$ is referred to appendix $[\mathrm{D}$ It is instructive to compare the evolution of the length scale and aggregation tendencies for the same simulation (see Figure 5 b and Figure $4 \mathrm{e}$ of Wing and Cronin [2016] for the evolution of the aggregation rates in time, calculated from the MSE spectrum and spatial anomalies, respectively). The longwave and shortwave aggregation tendencies are always positive, the advective aggregation tendency always negative, and the surface flux aggregation tendency transitions from positive to negative rates. Note that the amplitude of the diabatic spectral tendencies $\left\langle\dot{\varphi}_{i}\right\rangle$ (in units $\mathrm{J}^{2} \mathrm{~m}^{-4} \mathrm{~s}^{-1}$ ) increases less rapidly than the amplitude of the MSE spectrum $\langle\varphi\rangle$ (in units $\mathrm{J}^{2} \mathrm{~m}^{-4}$ ) as convection organizes, explaining why the amplitude of the aggregation rates $\left\langle\dot{\varphi}_{i}\right\rangle /\langle\varphi\rangle$ (in inverse time units) decreases with time.

Figure 6 summarizes the length scale and aggregation tendencies in both the growth phases and the mature phases of all twenty simulations. The horizontal axis represents the expansion rate $\left(\dot{L}_{i} / L\right)$, with a stretching effect to the right and a shrinking effect to the left of the zero line. The vertical axis represents the aggregation rate $\left(\left\langle\dot{\varphi}_{i}\right\rangle /\langle\varphi\rangle\right)$, with an aggregating effect above and a disaggregating effect below the zero line. Using the position of each MSE flux in $\left(\dot{L}_{i} / L,\left\langle\dot{\varphi}_{i}\right\rangle /\langle\varphi\rangle\right)$ space, we can extend our observations from the $300 \mathrm{~K}$ CAM-radiation long channel simulation to different sea surface temperatures, radiation schemes and geometries:

1. The longwave flux (blue markers) has positive length scale and aggregation rates during the first month across all simulations.

2. The shortwave flux (orange markers) has negative length scale and positive aggregation rates at all time across all simulations. We see a sharp dependence on surface temperature: rates for warm surface temperature simulations are significantly smaller than their cold surface temperature counterparts.

3. During the growth phase, the surface enthalpy flux (green markers) has large positive expansion and aggregation rates. During the mature phase, both rates become negative, with two notable exceptions. The mechanism denial experiments in which radiation is homogenized (MD RAD) maintain positive expansion and aggregation rates from surface fluxes in the mature phase, consistent with the weak aggregation of convection (see Figure 1 1 ). In the square experiments, the aggregation rate becomes negative while the expansion rate remains positive.

4. Finally, the advective flux (pink markers) exhibits the largest variability. In most simulations, advection shrinks $L$ in the growth phase and damps the convective aggregation at all times. It has the opposite effect on $L$ in the simulations with hottest sea surface temperature, as it can stretch $L$ during the mature phase. Both SQ and BSQ simulations have stretching yet disaggregating advective tendencies during the first month of the simulation. In the BSQ simulation, the surface enthalpy fluxes are responsible for balancing out the large advective stretching rate $\dot{L}_{\text {adv }} / L$ in the mature phase, since $\dot{\varphi}_{\text {adv }}$ homogenizes small scales faster than large scales in the BSQ geometry.

The last two sections investigate the sign and amplitude of aggregation and expansion rates from radiative and surface enthalpy fluxes. 


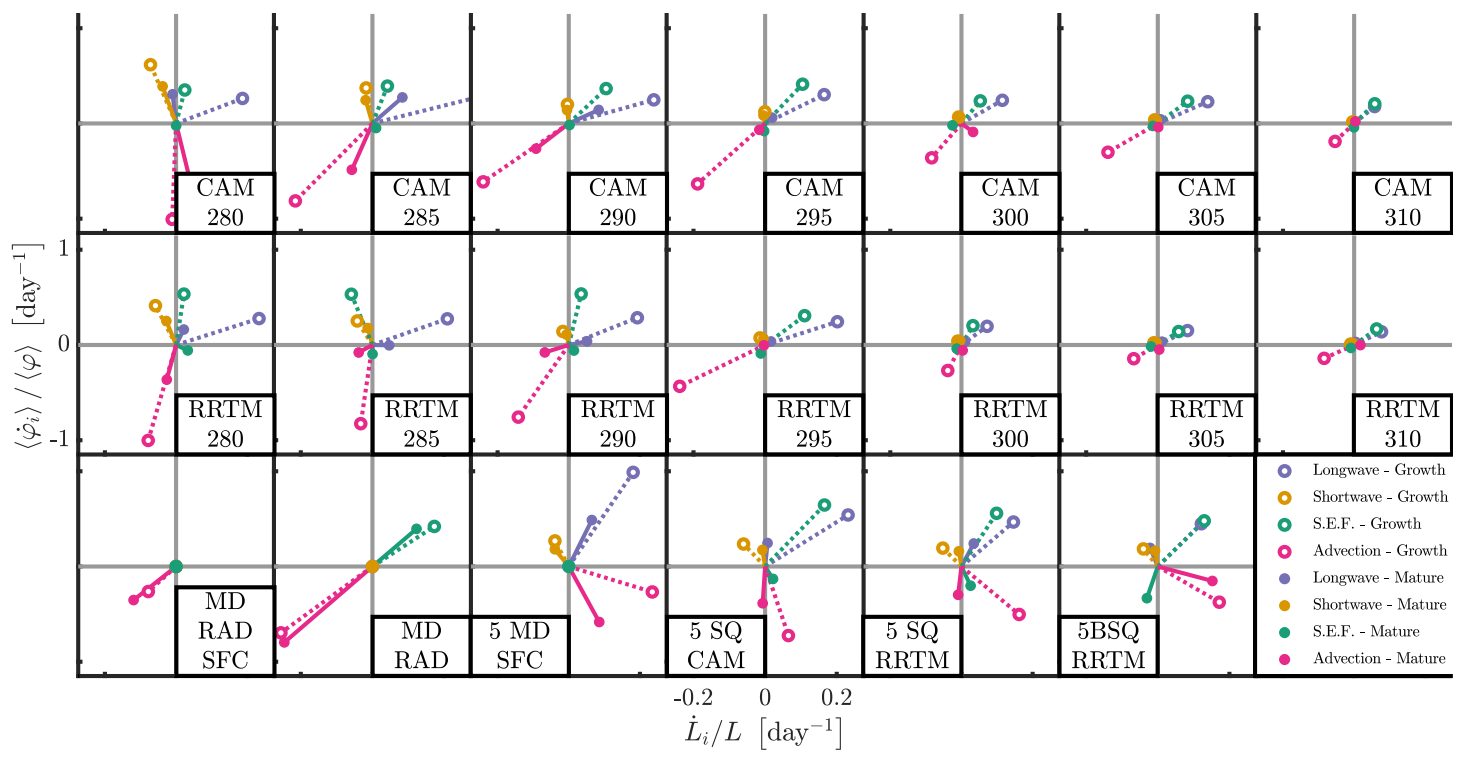

FIGURE 6 Representation of the twenty experiments in $\left(\dot{L}_{i} / L,\left\langle\dot{\varphi}_{i}\right\rangle /\langle\varphi\rangle\right)$ space. The aggregation and expansion rates have been time-averaged over the first month (Growth phase, depicted with dotted lines and empty circles) or later months (Mature phase, depicted with full lines and filled circles) of each experiment. Colors indicate different MSE fluxes: Longwave (blue), Shortwave (orange), Surface enthalpy flux (green) and advective flux (pink). All subplots use the same scale for both axes. 


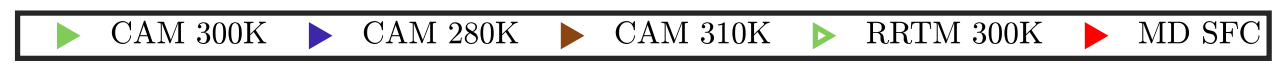

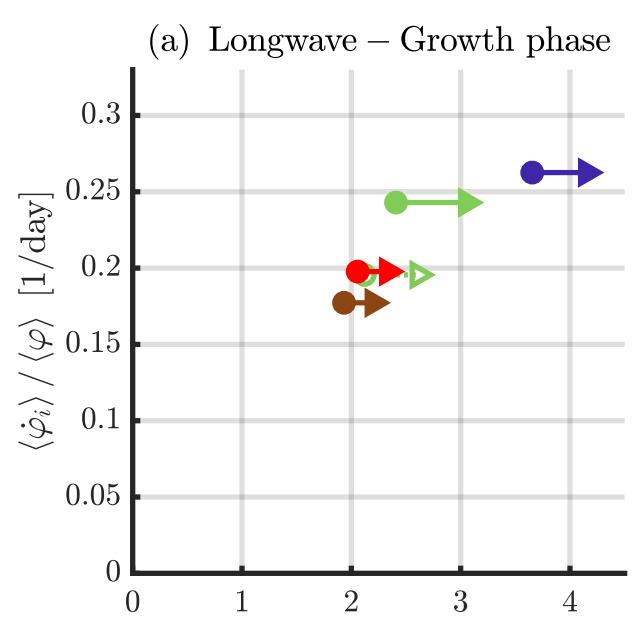

(c) Shortwave - Growth phase

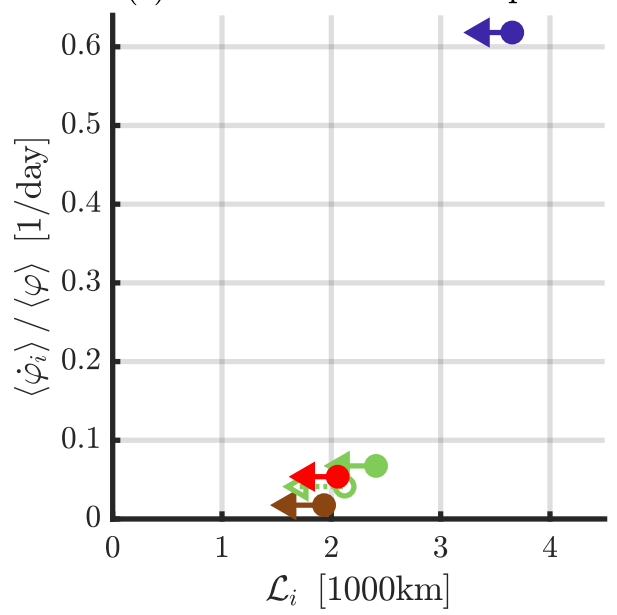

(b) Longwave - Mature phase

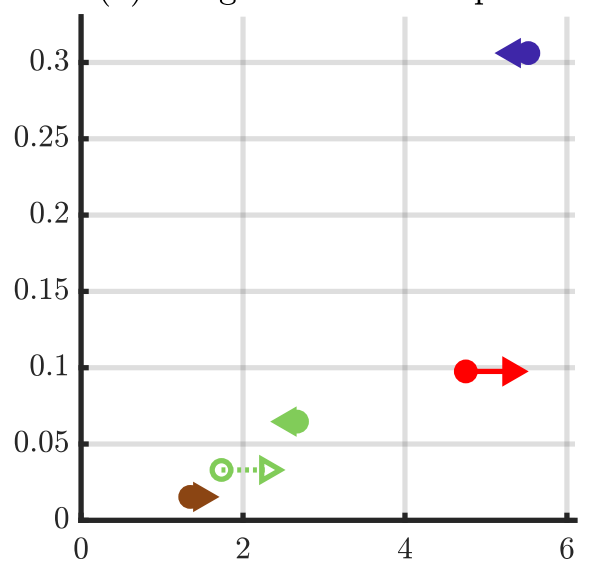

(d) Shortwave - Mature phase

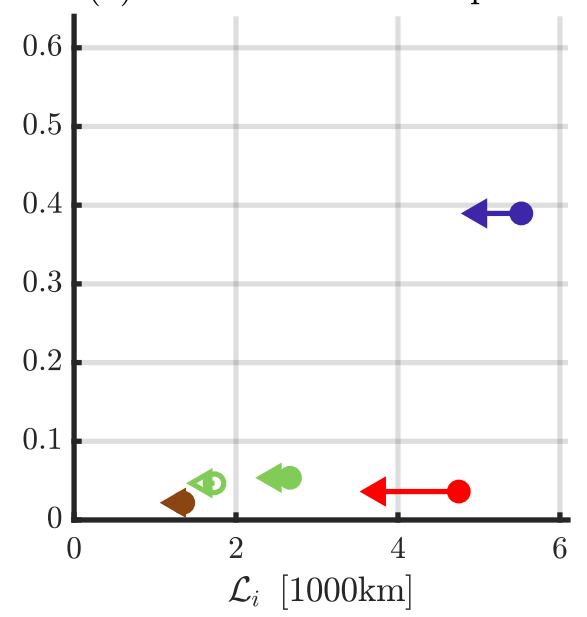

FIGURE 7 Representation of five characteristic experiments in $\left(\mathcal{L}_{i},\left\langle\dot{\varphi}_{i}\right\rangle /\langle\varphi\rangle\right)$ space. The longwave and shortwave rates have been time-averaged over the growth phases ((a) and (c) respectively) and mature phases ((b) and (d) respectively) of each simulation. The arrows go from the time-averaged convective length scale $L$ (represented with a circle) to the radiative length scale $\mathcal{L}_{i}$, and represent the length scale growth of each experiment (an arrow to the right corresponds to $\dot{L}_{i}>0$ ). The color code is that of Figure 4 
Since the radiative aggregation tendencies are positive across all simulations $\left(\left\langle\dot{\varphi}_{\mathrm{Iw}}\right\rangle>0\right.$ and $\left\langle\dot{\varphi}_{\mathrm{sw}}\right\rangle>0$, except during the first week of some cold simulations), radiation acts to pull the length scale of aggregation towards the radiative length scale (first line of Table 2. On the one hand, we find that the longwave length scale $\mathcal{L}_{\text {Iw }}$ usually exceeds the convective aggregation length scale $L$ during the growth phase, as shown in panels 7 . Therefore, the longwave tendency in the growth phase forces $L$ towards a larger scale $\mathcal{L}_{\mathrm{lw}}$, and $L_{\mathrm{iw}}>0$ across all simulations and phases as shown by the right-pointing arrows in the top-left panel. Even if the clear-sky longwave tendency contributes up to a third of the aggregation tendency $\left\langle\dot{\varphi}_{\mathrm{I}}\right\rangle$ at low temperature, its expansion tendency is always small relative to the total expansion tendency because the scale of water vapor anomalies is very close to the MSE anomaly scale. This is not the case in the mature phase (Figure $7 \mathrm{7}$ ), during which the longwave length scale $\mathcal{L}_{\text {lw }}$ may be smaller than the MSE anomaly scale $L$, resulting in a shrinking according to the top-left case of Table 2 On the other hand, we find that the shortwave length scale $\mathcal{L}_{s w}$ is smaller than $L$ during both phases, as shown in panels 7 f and $7 \mathrm{~d}$. Therefore, the shortwave tendency forces $L$ towards a smaller scale $\mathcal{L}_{\mathrm{sw}}$ and $L_{\mathrm{sw}}<0$ across all simulations and phases. The shortwave expansion tendency $L_{\text {sw }}$ is also dominated by the "cloudy" contribution because the clear-sky shortwave diabatic scale is so close to the MSE anomaly scale. Although a full analysis of the interaction between cloud radiation and convection is beyond the scope of this paper, we speculate that the contrast in longwave and shortwave length scales occurs due to the relative roles and spatial power spectra of clouds at different heights. Spectral analysis reveals that the net longwave heating is more coherent with column ice water, while the net shortwave heating is more coherent with column liquid water and column water vapor, which can be seen in Figure 11 of Appendix[D These results suggest that the net longwave heating variability is primarily dictated by high clouds, while the net shortwave heating variability is primarily dictated by low and mid-level clouds. High clouds are produced primarily by outflow from deep convection, which can spread far from the scattered convective cores, while low clouds are produced primarily by smaller-scale shallow-convective processes, giving us a first hint as to why $\mathcal{L}_{\mathrm{sw}}<L<\mathcal{L}_{\text {lw }}$. We refer the reader to section 6.2 of Bretherton and Khairoutdinov 2015 for a complementary discussion on this topic. 


\subsection{Surface flux rates}
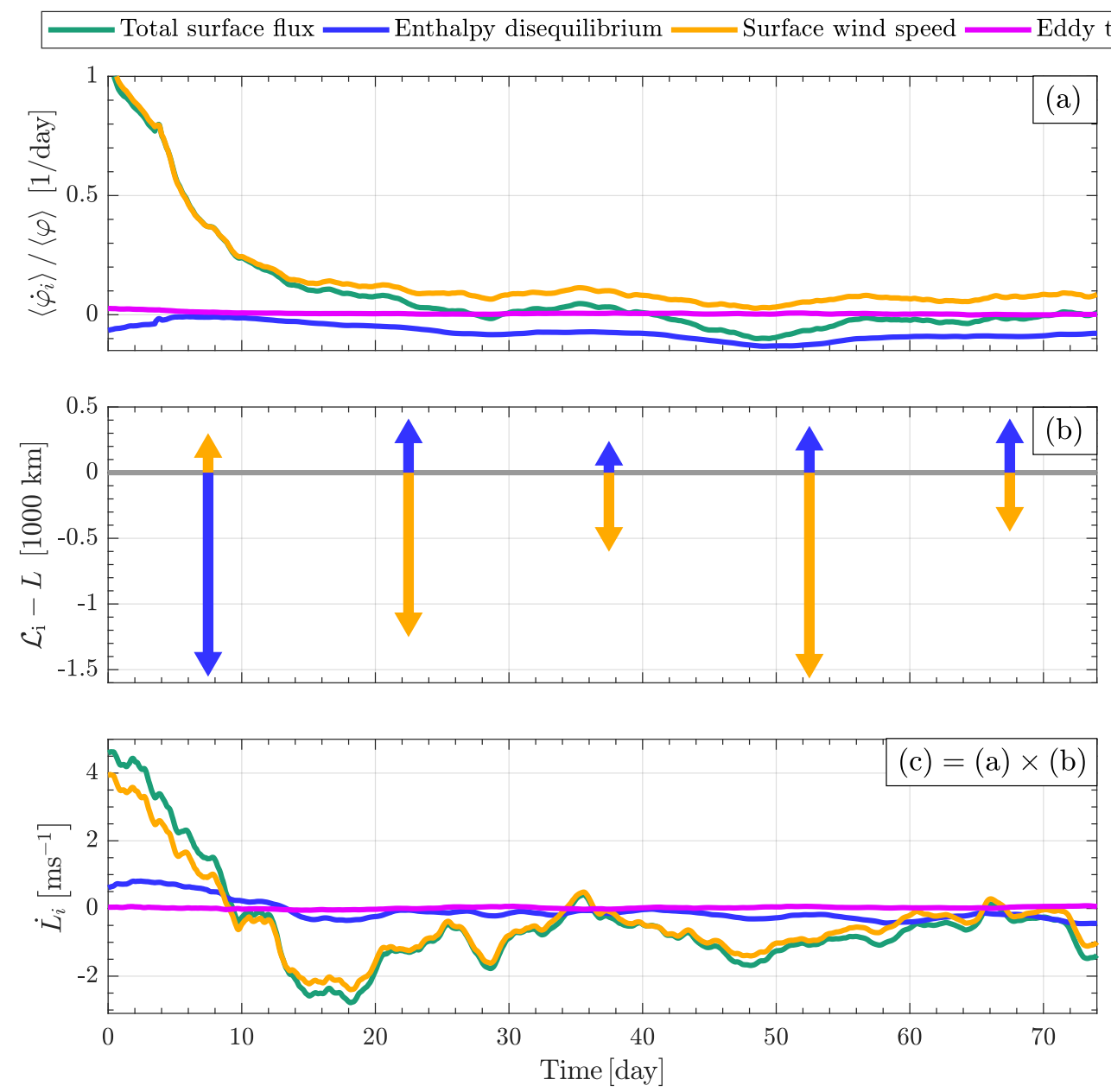

FIGURE 8 Decomposition of the surface flux aggregation rate (a), difference between diabatic length scale and MSE anomaly scale (b) and expansion tendency (c) for the LC300 CAM experiment. Panels (a) and (c) use a running time-average of 1 week while panel (b) shows length scale differences computed using 15 day time-averages. According to equation 15 the expansion rate (c) can be obtained by multiplying the aggregation rate (a) by the length scale difference (b). 
At first glance, the surface flux expansion tendency $L_{\text {sf }}$ is more challenging to interpret. The aggregation tendency changes sign across time and length scales, preventing us from using $\mathcal{L}_{\text {sf }}$ as a tool to interpret $\dot{L}_{\text {sf }}$. Following section 5.2 of Wing and Emanuel [2014], we decompose the surface enthalpy flux spatial anomaly into 3 contributions. Assuming a standard aerodynamic formula for the surface enthalpy flux, it can be written as the product of two factors:

$$
\mathrm{SF}=\rho C_{k} U_{s} \times \Delta k,
$$

where $\mathrm{SF}$ is the total surface enthalpy flux (in $\mathrm{W} \mathrm{m}^{-2}$ ), $\rho$ is the near-surface air density (in $\mathrm{kg} \mathrm{m}^{-3}$ ), $C_{k}$ is the surface enthalpy exchange coefficient, $U_{s}$ is the surface wind speed (in $\mathrm{m} \mathrm{s}^{-1}$ ) and $\Delta k$ is the air-sea enthalpy disequilibrium. We introduce the spatial-averaging operator \langle\rangle$_{\boldsymbol{x}}$ (see equation 33 in appendix A.2 and denote spatial anomalies with primes ('). By decomposing the two factors in equation 18 into their spatial means and anomalies, we decompose $\mathrm{SF}^{\prime}$ into 3 terms:

$$
\begin{aligned}
\mathrm{SF}^{\prime} & \stackrel{\text { def }}{=} \mathrm{SF}-\langle\mathrm{SF}\rangle_{\boldsymbol{x}} \\
& =\underbrace{\left(\rho C_{k} U_{s}\right)^{\prime}\langle\Delta k\rangle_{\boldsymbol{x}}}_{\text {Surface wind speed }}+\underbrace{\left\langle\rho C_{k} U_{s}\right\rangle_{\boldsymbol{x}} \Delta k^{\prime}}_{\text {Enthalpy disequilibrium }}+\underbrace{\left(\rho C_{k} U_{s}\right)^{\prime} \Delta k^{\prime}-\left\langle\rho C_{k} U_{s}\right\rangle_{\boldsymbol{x}}\langle\Delta k\rangle_{\boldsymbol{x}}}_{\text {Eddy term }} .
\end{aligned}
$$

We apply this same decomposition to the aggregation and expansion surface flux rates, which is shown in Figure 8 Figure 8 a shows that the wind and disequilibrium aggregation rates are each consistent in sign for the whole simulation, while the eddy aggregation rate is negligible at all times. Convectively active zones exhibit more convective gustiness, making the wind aggregation tendency always positive. Meanwhile, convectively active zones are moister and have a smaller enthalpy disequilibrium, keeping the disequilibrium aggregation tendency negative. During the growth phase, the wind aggregation rate dominates over the two other aggregation rates, and the total aggregation rate is positive. During the mature phase, the two aggregation rates oppose each other and the total aggregation rate switches from positive to negative, as the disequilibrium rate becomes more and more negative. This finding is consistent with results from square-domain non-rotating simulations presented in this manuscript and panel $5 \mathrm{~d}$ of Wing et al. [2016]; panel 5c of Wing et al. [2016] suggests that the positive surface wind speed feedback dominates for all times in simulations of rotating convection (in which tropical cyclones form). We plot the length scale factor of equation 15 in Figure 8 p. Since the wind and disequilibrium aggregation rates are single-signed, the interpretation of their diabatic length scales $\mathcal{L}_{i}$ is easier. The evolution of $L_{\text {wind }}$ (depicted in Figure 8) follows from the first line of table 2 For $t<12$ day, the surface wind speed component of the surface flux aggregates convection at larger scales and stretches $L$ (analogous to $L_{\text {iw }}$ ), while for $t>12$ day, it aggregates convection at smaller scales and shrinks $L$ (analogous to $L_{\mathrm{sw}}$ ). The surface wind speed component of the surface flux includes contributions from both the large-scale circulations that develop throughout the simulation, and convective gustiness. We find that $\mathcal{L}_{\text {wind }}$ quickly becomes smaller than $L$ as convection aggregates and $L$ grows (Orange arrows in Figure $8 \mathrm{p}$ ), suggesting that convective gustiness dominates the surface wind speed spectral tendency $\dot{\varphi}_{\text {wind }}$. While both aggregation tendencies $\left\langle\dot{\varphi}_{\text {wind }}\right\rangle$ and $\left\langle\dot{\varphi}_{\text {dis }}\right\rangle$ have the same order of magnitude, the enthalpy disequilibrium flux acts at the scale of MSE anomalies. Its diabatic length scale $\mathcal{L}_{\text {dis }}$ is then close to $L$, and the amplitude of its expansion tendency is significantly smaller than the surface wind expansion tendency $\left(\left|L_{\text {dis }}\right| \ll\left|L_{\text {wind }}\right|\right)$. Therefore, the evolution of the wind expansion tendency $L_{\text {wind }}$ explains the evolution of the surface flux expansion tendency $L_{\text {sf }}$ to first order; the disequilibrium tendency slightly stretches $L$ during the growth phase and slightly damps it during the mature phase. 


\section{CONCLUSION}

We have developed a budget that relates the evolution of the convective aggregation length scale (defined in section 2.1 to the vertically-integrated MSE fluxes (section 2.2. We have used a set of cloud-permitting three-dimensional simulations (section 2.3) to evaluate (section 2.4 and interpret (section 2.5) the different terms of this budget across different experiments. In section 3.1 we have divided the evolution of convective aggregation into two phases: a growth phase during which convection self-aggregates, and a mature phase during which the size of moist and dry regions depends on the characteristics of the simulation. In section 3.2 we have characterized the aggregation and expansion tendencies across experiments, and have investigated the values of the radiative and surface flux rates in sections 3.3 and 3.4. The surface temperature dependence of the expansion rates is summarized in Figure 9

1. The first-month-averaged longwave expansion rates $\left(L_{\mathrm{lw}} / L\right)$ decrease with surface temperature following the decrease in longwave aggregation rates $\left\langle\dot{\varphi}_{\mid \mathrm{w}}\right\rangle /\langle\varphi\rangle$. The aggregation rate decrease comes from a decrease in column ice, as well as an increase in the degree of aggregation with surface temperature. Aggregation rates are higher for the CAM than RRTM radiation scheme, which can be traced back to larger net cloudy radiative heating aggregation rates when the CAM radiation scheme is used. In the long-channel setting, the net cloudy longwave heating is the crucial ingredient for convection to aggregate, as confirmed by the low degree of aggregation in the MD RAD and MD RADSFC experiments. The mature-phase-averaged longwave expansion rates $\left(\dot{L}_{\mathrm{lw}} / L\right)$ also decrease with surface temperature, and may even become slightly negative for the CAM radiation scheme, as the longwave diabatic length scale $\mathcal{L}_{\text {lw }}$ becomes smaller than the scale $L$ of MSE anomalies.

2. The shortwave expansion rates $\left(L_{s w} / L\right)$ become less negative as surface temperature increases, consistent with the decrease in shortwave aggregation rates with surface temperature. The net shortwave heating is always dominated by its clear-sky component; as temperature increases, this component becomes more and more prevalent. Since the clear-sky shortwave flux acts at the MSE anomaly scale to good approximation, it does not have any shrinking effect and $L_{\text {sw }}$ becomes closer and closer to zero.

3. The initial surface enthalpy flux expansion rate $\left(\dot{L}_{\mathrm{sf}} / L\right)$ increases with surface temperature, mostly due to an increase in the wind-induced component of the latent heat flux expansion rate with surface temperature. That increase comes from a change in the spectral shape of the surface flux spectral tendency $\dot{\varphi}_{\text {sf }}$, since the firstmonth-averaged surface enthalpy flux aggregation rates $\left\langle\dot{\varphi}_{\text {sf }}\right\rangle /\langle\varphi\rangle$ decrease with surface temperature: In Figure 6 the green empty circles shift to the bottom-right of $\left(\dot{L}_{\mathrm{sf}} / L,\left\langle\dot{\varphi}_{\mathrm{sf}}\right\rangle /\langle\varphi\rangle\right)$ space as surface temperature increases. The mature-phase-averaged surface enthalpy flux expansion rate $\left(L_{\text {sf }} / L\right)$ switches from positive at low surface temperatures, for which the enthalpy disequilibrium component prevails, to negative at higher surface temperatures because of the wind component's spectral peak below the MSE anomaly scale $L$ (see Appendix 4 . The strong shrinking effect of surface enthalpy fluxes is what prevents MSE power from being transferred to the longest wavelength in the long-channel setting, as confirmed by the MD SFC experiment exhibiting the largest $L$ that keeps increasing after three months of simulation.

4. Finally, the advective flux always has a shrinking effect during the first month, except in square domain geometry simulations where it has a stretching effect because it disaggregates convection at small scales (see Figure6). It shifts from shrinking to stretching with surface temperature during the mature phase as it homogenizes MSE at smaller and smaller scales.

For each flux, we represent the expansion rate's temporal variability with error bars, which heights equal three standard deviations divided by the sample size's square root. If the temporal variability had a Gaussian distribution, these error 
bars would be equal to three times the root-mean-squared error on the time-averaged expansion rates. The largest variability arises from the advective and longwave expansion rates during the first month of the simulations, with the advective rate potentially becoming positive as its spectral peak shifts to smaller wavelengths. Note that the dependence of the expansion tendencies with surface temperature is more visible if we use metrics that give more weight to smaller wavelengths, such as the integral scale studied in Appendix

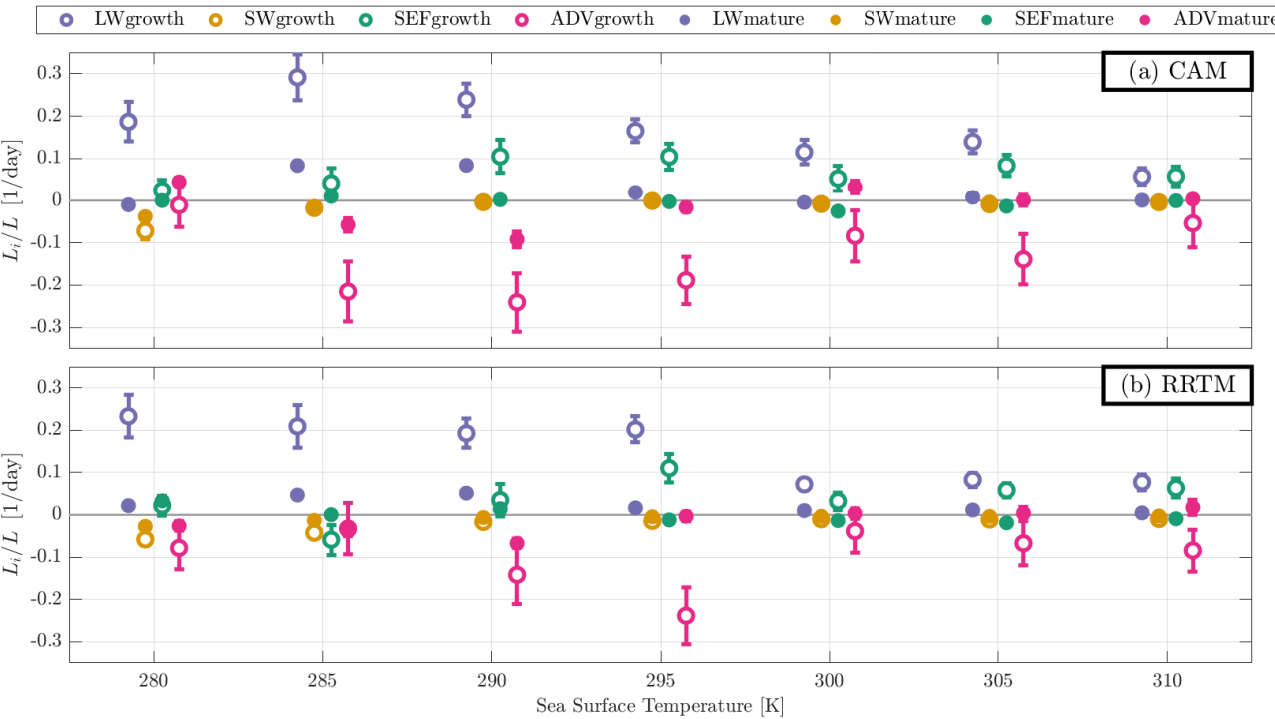

FIGURE 9 Expansion rate (in 1/day) versus sea surface temperature for the long-channel experiments using the (a) CAM and (b) RRTM radiation schemes. The rates are time-averaged during the first month (Growth phase, empty circles) and later months (Mature phase, filled circles) of each simulation; we use error bars to indicate three standard deviations normalized by the sample size's square root. Each color corresponds to a MSE flux: Longwave (blue), Shortwave (orange), Surface enthalpy flux (green) and Advective flux (pink).

By relating local physical processes to large-scale convective organization, this budget emphasizes the need to better understand individual spectral tendencies $\dot{\varphi}_{i}(\boldsymbol{k})$. We still lack a fundamental physical explanation for the value of cloud radiative spectral tendencies, which are key players in setting the size and intensity of moist and dry regions. Furthermore, the influence of radiative tendencies on the organization of convection in the real world remains a topic of active research, as inhomogeneous boundary feedbacks and external shear make the physical processes harder to distinguish in practice. The framework presented here focuses on the expansion tendencies rather than a static aggregated state, which is a step towards diagnosing clustering feedbacks in the real world where the constantly-evolving size of moist and dry regions challenges the idea of a single MSE anomaly scale. Although the present manuscript focuses on MSE, our framework can be generalized to understand the dominant scale of any conserved variable, e.g. the scale of initial wind anomalies using the turbulent kinetic energy and available potential energy budgets [Yang 2018]. In cloud-permitting simulations, a framework to decompose spectral advective tendencies into their deep, shallow and diabatically-driven components could also help bridge this gap by isolating the indirect role of diabatic processes through the circulation they induce (e.g. Holloway and Woolnough [2016]). Finally, we have explored the surface temperature dependence of convective organization; making the surface temperature interactive will be a necessary 
step to connect cloud-permitting simulations with the real world, especially given how the interaction between the atmosphere and a shallow slab-ocean affects the organization of convection (e.g. Hohenegger and Stevens [2018] for a Convection-Permitting study and Coppin and Bony [2017] for a recent General Circulation Model study). Overall, our work underscores the need to observe and simulate surface fluxes, radiative and advective fluxes across the $1 \mathrm{~km}-1000 \mathrm{~km}$ range of scales to better understand the characteristics of turbulent moist convection.

\section{ACKNOWLEDGEMENTS}

Tom Beucler is supported by NSF grant AGS-1520683, while Timothy Cronin is supported by NSF grant AGS-1623218. We thank Allison Wing for review and assistance regarding the present manuscript, as well as for providing seventeen of the twenty cloud-permitting simulations analyzed in this paper, including the unpublished big square-domain simulation; these simulations were run on the Yellowstone computing cluster provided by the National Center for Atmospheric Research's Computational and Information Systems Laboratory, sponsored by the National Science Foundation. The three new mechanism denial cloud-permitting simulations were run on the Engaging computing cluster at MIT. We thank Marat Khairoutdinov for providing the authors with SAM. The authors thank Christopher Holloway and two anonymous reviewers for detailed comments that helped improve the quality of the manuscript, Sydney Sroka and Nicholas Lutsko for proofreading, and Tristan Abbott, Glenn Flierl, Raffaele Ferrari and Santiago Benavides for scientific discussions that helped the project. The source code and data used to produce the figures can be found at https: //github.com/tbeucler/2018_L_budget_convective_aggregation

\section{A. Alternative definitions of $L$}

\section{A.1. Comparison of spectrally-defined scales to the auto-correlation length scale}

In this article, we adopt the intuitive definition 2 of the convective aggregation length scale. This definition can be generalized to $L \stackrel{\text { def }}{=} 2 \pi \sqrt{n}\left\langle\|\boldsymbol{k}\|^{p} \varphi\right\rangle^{1 / q}\left\langle\|\boldsymbol{k}\|^{p+q} \varphi\right\rangle^{-1 / q}$, with the choice of $(p, q)$ determining which parts of the MSE spectrum are given the most weight. In this appendix, we compare three cases of this general definition:

1. The power spectrum-weighted-averaged wavelength, which is the definition used in the main text:

$$
(p, q)=(-1,1) \Rightarrow L \stackrel{\text { def }}{=} \frac{2 \pi \sqrt{n}}{\langle\varphi\rangle}\left\langle\frac{\varphi}{\|k\|}\right\rangle,
$$

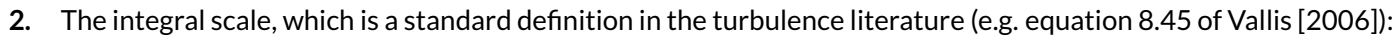

$$
(p, q)=(1,-1) \Rightarrow K^{-1} \stackrel{\text { def }}{=} \frac{2 \pi \sqrt{n}\langle\varphi\rangle}{\langle\|k\| \varphi\rangle},
$$

3. The geometric mean of the two previous definitions:

$$
(p, q)=\left(-\frac{1}{2}, \frac{1}{2}\right) \Rightarrow \sqrt{L K^{-1}} \stackrel{\text { def }}{=} 2 \pi \sqrt{n} \sqrt{\frac{\langle\varphi /\|\boldsymbol{k}\|\rangle}{\langle\|\boldsymbol{k}\| \varphi\rangle}} .
$$

A fourth plausible choice of length scale in our comparison is the spatial auto-correlation scale of the MSE field $L_{\varphi}(t)$, defined as the e-folding length of the autocorrelation function (e.g. section 8.10 of Dunn 2005]). In one-dimension, 
467

$L_{\varphi}(t)$ can be defined from the normalized inverse Fourier transform $\phi$ of the MSE power spectrum $\varphi$ as:

$$
L_{\varphi}(t) \stackrel{\text { def }}{=} \min _{x}\{x: \phi(x, t)<\exp (-1)\}
$$

468

$$
\phi(x, t) \stackrel{\text { def }}{=} \frac{\int_{k_{0}}^{k_{N}} \exp (i k x) \varphi(k, t) d k}{\int_{k_{0}}^{k_{N}} \varphi(k, t) d k} .
$$

Note that the definition of $L_{\varphi}$ can yield unrealistic large values when the power spectrum $\varphi$ is concentrated at low wave numbers, which is the case during the mature phase of our simulations. In that case, we set unrealistic large values, typically greater than the domain size, to their nearest realistic neighbor. Furthermore, if the oscillations of $\phi$ in $x$ cross the hard threshold $\exp (-1)$ rapidly, there is ambiguity in the physical meaning of $L_{\varphi}$, even if we choose the smallest solution and define a unique value for $L_{\varphi}$. For these reasons, we believe $L_{\varphi}$ is too ill-defined to be used as a systematic metric when studying the physics of convective aggregation in our simulations. Nevertheless, $L_{\varphi}$ is a standard metric for the scale of spatial anomalies, and we compare it to to $L, K^{-1}$ and $\sqrt{L K^{-1}}$ for our reference LC300CAM simulation in Figure A1. 


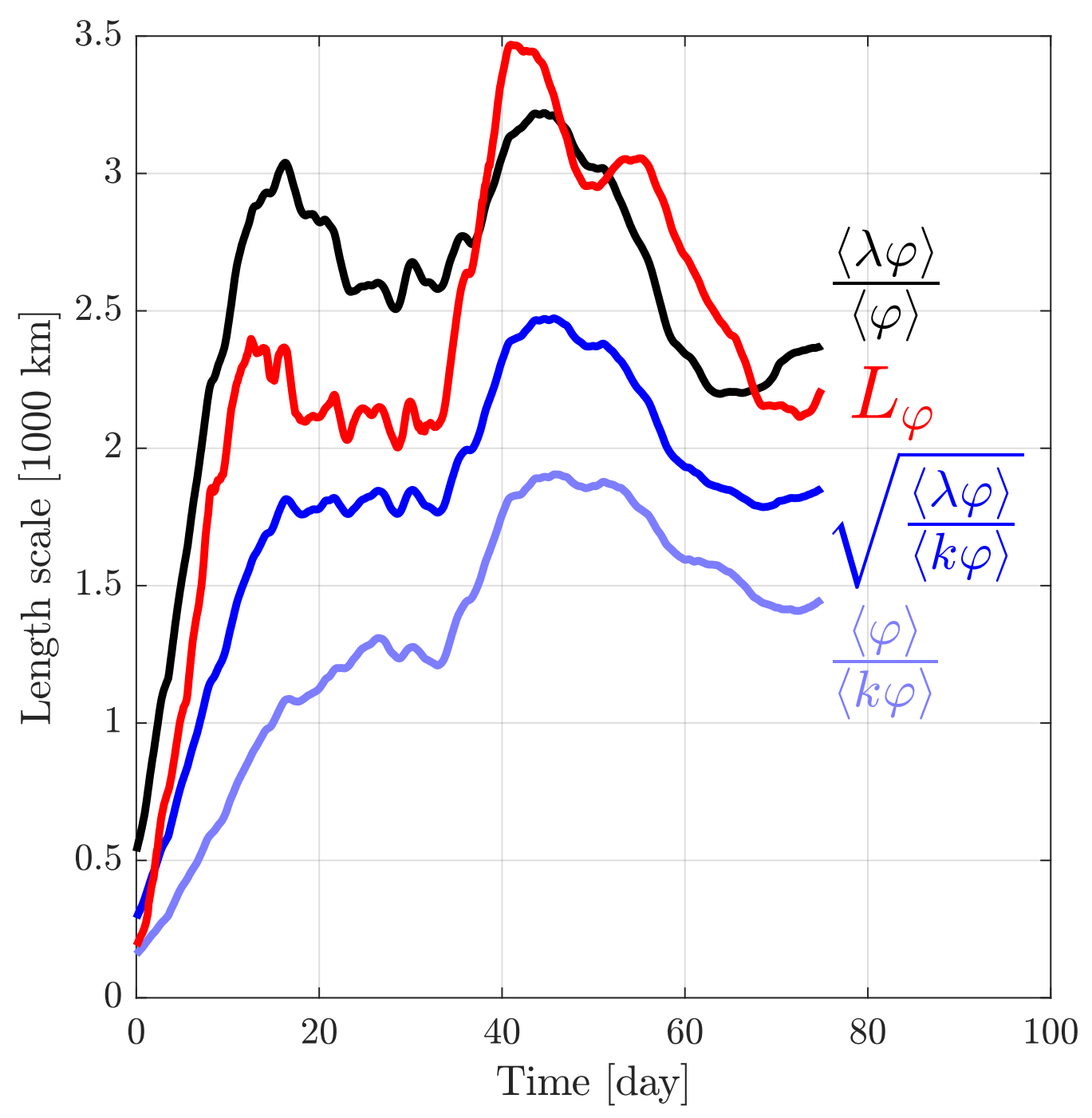

FIGURE 10 (Black line) $L(t)$, (Red line) $L_{\varphi}(t)$, (Blue line) $\sqrt{L K^{-1}}(t)$ and (Sky blue line) $K^{-1}(t)$ (in $1000 \mathrm{~km}$ ) versus time (in days) for the reference LC300CAM simulation.

Because $L$ gives the most weight to the longest wavelengths, it is the largest metric for the scale of MSE spatial anomalies. Furthermore, it is the closest metric to the auto-correlation scale $L_{\varphi}$. The two other metrics both capture the low-frequency time-evolution of the auto-correlation scale (growing-decaying-growing-decaying), suggesting that their budgets may contribute to our understanding of the MSE spectrum evolution. Therefore, we derive a budget for the integral MSE scale $K^{-1}(t)$ in the next sub-section A.2. One notable shortcoming of the integral scale $K^{-1}$ arises in squaredomain geometries and non-aggregated simulations. Because the spectral slope in square domain geometries oscillates 
494

around $\varphi \sim\left\|\sqrt{k^{2}+l^{2}}\right\|^{-3}$ in the SQ and BSQ simulations, where $(k, l)$ are the components of the two-dimensional wave vector $\boldsymbol{k}$, the average:

$$
\langle\|\boldsymbol{k}\| \varphi\rangle \stackrel{\operatorname{def}}{=} \frac{\int_{k_{0}}^{k_{N}} d k \int_{l_{0}}^{I_{N}} d l \times \sqrt{k^{2}+l^{2}} \times \varphi(k, l)}{\int_{k_{0}}^{k_{N}} d k \int_{l_{0}}^{I_{N}} d l \times \varphi(k, l)}
$$

may depend on the domain's resolution through $\left(k_{N}, I_{N}\right)$. The same issue arises in non-aggregated simulations, where the spectral slope $s$ of $\varphi \sim k^{-s}$ may be smaller than 2 . This dependence limits the physical understanding that we can obtain from the $K^{-1}$-budget for the LC CAM, LC RRTM, and MD SFC experiments.

\section{A.2. Budget for the integral moist static energy scale}

This section derives a budget for the integral MSE scale $K^{-1}$, similar to the convective aggregation length scale derived in section 2.2.3. We start by deriving a budget for $K \stackrel{\text { def }}{=}\langle\|\boldsymbol{k}\| \varphi\rangle /\langle\varphi\rangle$, by following the exact steps of section 2.2.3 and substituting $\lambda \mapsto\|\boldsymbol{k}\|$. By analogy to equations 13, 15 and 16, we can write the evolution of $K$ as a sum of diabatic tendencies $\dot{K}_{i}$, defined as the product of the aggregation tendency with a growth length scale factor towards the diabatic integral wave number $\mathcal{K}_{i}$ :

$$
\frac{\partial K}{\partial t}=\dot{K}_{\mathrm{lw}}+\dot{K}_{\mathrm{sw}}+\dot{K}_{\mathrm{sef}}+\dot{K}_{\mathrm{adv}}
$$

$$
\forall i, \dot{K}_{i}=\frac{\left\langle\dot{\varphi}_{i}\right\rangle}{\langle\varphi\rangle}\left(\mathcal{K}_{i}-K\right) \mid \mathcal{K}_{i} \stackrel{\text { def }}{=} \frac{2 \pi \sqrt{n}\left\langle\|\boldsymbol{k}\| \dot{\varphi}_{i}\right\rangle}{\left\langle\dot{\varphi}_{i}\right\rangle}
$$

The budget for the integral scale $K^{-1}$ can be derived by using a logarithmic derivative:

$$
\frac{1}{K^{-1}} \frac{\partial K^{-1}}{\partial t}=-\frac{1}{K} \frac{\partial K}{\partial t} \Rightarrow \frac{\partial K^{-1}}{\partial t}=\sum_{i=\mathrm{lw}, \mathrm{sw}, \mathrm{sef}, \mathrm{adv}}\left(K^{\dot{-1}}\right)_{i}
$$

$$
\forall i,\left(K^{-1}\right)_{i}=\underbrace{\frac{\left\langle\dot{\varphi}_{i}\right\rangle}{\langle\varphi\rangle}}_{\text {Aggregation rate }} \overbrace{K^{-1}\left(1-\frac{K^{-1}}{\mathcal{K}_{i}^{-1}}\right)}^{\text {Length scale factor }} .
$$

The length scale factor in equation 29 is analogous to its counterpart in equation 16 because each flux (i) forces the MSE integral scale $K^{-1}$ towards its intrinsic integral scale $\mathcal{K}_{i}^{-1}$, but it differs in its functional form: the growth is now logistic rather than exponential. This modifies the interpretation of the diabatic integral scale $\mathcal{K}_{i}^{-1}$ as summarized in Table 3 analogous to Table 2 . 


\begin{tabular}{|c|c|c|c|}
\hline & Small scale: $0<\mathcal{K}_{i}^{-1}<K^{-1}$ & Large-scale: $\mathcal{K}_{i}^{-1}>K^{-1}$ & Negative scale: $\mathcal{K}_{i}^{-1}<0$ \\
\hline \hline Aggregation: $\left\langle\dot{\varphi}_{i}\right\rangle>0$ & Shrinking: $\left(K^{-1}\right)_{i}<0$ & Stretching: $\left(K^{-1}\right)_{i}>0$ & "Super stretching": $\left(K^{-1}\right)_{i}>0$ \\
\hline Disaggregation: $\left\langle\dot{\varphi}_{i}\right\rangle<0$ & Stretching: $\left(K^{-1}\right)_{i}>0$ & Shrinking: $\left(K^{-1}\right)_{i}<0$ & "Super shrinking": $\left(K^{-1}\right)_{i}<0$ \\
\hline
\end{tabular}

TA B LE 3 Guide for interpreting equation 29 which relates the integral scale tendencies $\left(K^{-1}\right)_{i}$ to the aggregation tendencies $\left\langle\dot{\varphi}_{i}\right\rangle /\langle\varphi\rangle$ and the diabatic integral scales $\mathcal{K}_{i}^{-1}$.

When a flux $\dot{H}_{i}$ acts at a scale $\mathcal{K}_{i}^{-1}$ that is equal to the MSE integral scale $K^{-1}$, then it does not affect the scale of convective activity and $\left(K^{-1}\right)_{i}=0$; the singular case $\mathcal{K}_{i}^{-1}=0$ would correspond to an infinite integral scale tendency $\left(K^{-1}\right)_{i}$. When the scale $\mathcal{K}_{i}^{-1}$ is negative (possible because the spectral tendencies $\dot{\varphi}_{i}$ can be double-signed), there are two possible cases:

1. If the aggregation tendency $\left\langle\dot{\varphi}_{i}\right\rangle$ is positive, then the stretching $\left(K^{-1}\right)_{i}>0$ is larger than the stretching for any positive $\mathcal{K}_{i}^{-1}$ greater than the scale of aggregation (referred to as "Super stretching"):

$$
\left(K^{-1}\right)_{i}\left(\mathcal{K}_{i}^{-1}<0\right)>\left(K^{-1}\right)_{i}\left(\mathcal{K}_{i}^{-1}>K^{-1}\right)>0
$$

2. If the aggregation tendency $\left\langle\dot{\varphi}_{i}\right\rangle$ is negative, then the amplitude of the shrinking is larger than the amplitude of the shrinking for any positive $\mathcal{K}_{i}^{-1}$ smaller than the scale of aggregation (referred to as "Super shrinking"):

$$
\left(K^{-1}\right)_{i}\left(\mathcal{K}_{i}^{-1}<0\right)<\left(K^{-1}\right)_{i}\left(\mathcal{K}_{i}^{-1} \in\right] 0, K^{-1}[)<0
$$

These cases can only occur in a sustained context when a process acts to damp MSE variability over one range of spatial scales and amplify it across another range, and when the MSE spatial variance over the range of damped scales is maintained by other processes (so that its weighting in equation 11 does not vanish as time passes).

The qualitative conclusions regarding the role of each diabatic flux in setting the scale of MSE anomalies are insensitive to the choice of $L$ or $K^{-1}$ as a metric, except for the MD RAD, MD RAD+SFC, SQ and BSQ simulations for which $K$ depends on the simulation's resolution. The reader interested in a more quantitative comparison is referred to the article's MATLAB scripts: using the "User's choice" options, each figure of the main text can be remade using the budget for $K$ rather than for $L$.

\section{B. EquiVALENCE OF THE MOIST STATIC ENERGY VARIANCE AND POWER SPECTRUM BUDGETS}

First, consider two real-valued functions of position, $f(\boldsymbol{x})$ and $g(\boldsymbol{x})$ that are periodic with zero average on a rectangular domain extending from the origin $(\boldsymbol{x}=0)$ to $\left(\boldsymbol{x}=\boldsymbol{L}_{\mathrm{dom}}\right)$. We will prove that:

$$
\langle f g\rangle_{x}=\mathcal{N}\left\langle\operatorname{Re}\left(\widehat{f} \widehat{g}^{*}\right)\right\rangle_{\boldsymbol{k}},
$$


and spectral space:

$$
\langle X\rangle_{k} \stackrel{\operatorname{def}}{=} \frac{\int_{k_{0}}^{k_{N}} X(k) d k}{\int_{k_{0}}^{k_{N}} d k},
$$

where $\widehat{f}(\boldsymbol{k})$ and $g(\boldsymbol{k})$ are the spatial Fourier transforms of $f$ and $g$, defined in equation 5 and $\mathcal{N}$ a constant that depends on the size and resolution of the integration domain. We have introduced the averaging operators in physical space:

$$
\langle X\rangle_{\boldsymbol{x}} \stackrel{\text { def }}{=} \frac{\int_{0}^{L_{\mathrm{dom}}} X(\boldsymbol{x}) d \boldsymbol{x}}{\int_{0}^{L_{\mathrm{dom}}} d \boldsymbol{x}},
$$

where the spectral domain extends from the wavenumber with smallest modulus $k=k_{0}$ to the Nyquist wavenumber $k=k_{N}$. The proof can be done in four steps:

1. Assuming $(f, g)$ are square-integrable functions, we can prove Parseval's lemma regarding the scalar product of square integrable functions:

$$
\begin{aligned}
& \int_{0}^{L_{\mathrm{dom}}} f(\boldsymbol{x}) g(\boldsymbol{x}) d \boldsymbol{x}=\int_{0}^{\boldsymbol{L}_{\mathrm{dom}}} f(\boldsymbol{x}) g(\boldsymbol{x})^{*} d \boldsymbol{x} \\
& =\frac{1}{(2 \pi)^{n}} \int_{\mathbb{R}^{n}} d \boldsymbol{x} \int_{\mathbb{R}^{n}} d \boldsymbol{k} e^{-\ell \boldsymbol{k} \cdot \boldsymbol{x}} \widehat{f}(\boldsymbol{k}) \int_{\mathbb{R}^{n}} d \boldsymbol{k} / \boldsymbol{e}^{+\ell \boldsymbol{k} \cdot \boldsymbol{x}} \widehat{\boldsymbol{g}}(\boldsymbol{k} \prime)^{*} \\
& =\frac{1}{(2 \pi)^{n}} \int_{\mathbb{R}^{n}} \int_{\mathbb{R}^{n}} \int_{\mathbb{R}^{n}} d \boldsymbol{x} d \boldsymbol{k} d \boldsymbol{k} \prime \cdot \boldsymbol{e}^{\ell(\boldsymbol{k} \prime-\boldsymbol{k}) \cdot \boldsymbol{x}} \widehat{f}(\boldsymbol{k}) \widehat{g}(\boldsymbol{k} \prime)^{*} \\
& =\int_{\mathbb{R}^{n}} \int_{\mathbb{R}^{n}} d \boldsymbol{k} d \boldsymbol{k}^{\prime} \cdot \delta(\boldsymbol{k}-\boldsymbol{k}) \widehat{f}(\boldsymbol{k}) \widehat{\boldsymbol{g}}(\boldsymbol{k} \prime)^{*} \\
& =\int_{\mathbb{R}^{n}} d \boldsymbol{k} \widehat{f}(\boldsymbol{k}) \widehat{g}(\boldsymbol{k})^{*} \\
& =\int_{\mathbb{R}_{+}^{n}} d \boldsymbol{k} \widehat{f}(\boldsymbol{k}) \widehat{g}(\boldsymbol{k})^{*}+\int_{\mathbb{R}_{\underline{n}}^{\underline{n}}} d \boldsymbol{k} \widehat{f}(\boldsymbol{k}) \widehat{\boldsymbol{g}}(\boldsymbol{k})^{*}
\end{aligned}
$$

where we have introduced the Dirac delta function $\delta$ and the $n$-dimensional real number set $\mathbb{R}^{n}$.

2. Since the functions $(f, g)$ are real-valued, their Fourier transforms verify:

$$
\widehat{f}(-\boldsymbol{k})=\widehat{f}(\boldsymbol{k})^{*}, \widehat{\boldsymbol{g}}(-\boldsymbol{k})=\widehat{\boldsymbol{g}}(\boldsymbol{k})^{*}
$$

3. In the last line of step 1, we have broken the integral over $\mathbb{R}^{n}$ in two parts: an integral over the positive numbers $\left(\mathbb{R}_{+}^{n}\right)$ and an integral over the negative numbers $\left(\mathbb{R}_{-}^{n}\right)$. Using step 2 , we can change variables and transform the integral over $\mathbb{R}_{-}^{n}$ into an integral over $\mathbb{R}_{+}^{n}$ :

$$
\begin{aligned}
\int_{\mathbb{R}^{n}} d \boldsymbol{k} \widehat{f}(\boldsymbol{k}) \widehat{\boldsymbol{g}}(\boldsymbol{k})^{*} & =\int_{\mathbb{R}_{+}^{n}} d \boldsymbol{k} \widehat{f}(\boldsymbol{k}) \widehat{\boldsymbol{g}}(\boldsymbol{k})^{*}+\int_{\mathbb{R}^{n}} d \boldsymbol{k} \widehat{f}(-\boldsymbol{k})^{*} \widehat{\boldsymbol{g}}(-\boldsymbol{k}) \\
& =\int_{\mathbb{R}_{+}^{n}} d \boldsymbol{k} \widehat{f}(\boldsymbol{k}) \widehat{\boldsymbol{g}}(\boldsymbol{k})^{*}+\int_{\mathbb{R}_{+}^{n}} d \boldsymbol{k} \widehat{f}(\boldsymbol{k})^{*} \widehat{g}(\boldsymbol{k}) \\
& =2 \int_{\mathbb{R}_{+}^{n}} d \boldsymbol{k} \cdot \operatorname{Re}\left[\widehat{f}(\boldsymbol{k}) \widehat{g}(\boldsymbol{k})^{*}\right]
\end{aligned}
$$


533

4. The product $(f g)$ is periodic in $\boldsymbol{x}$ and its average is the same over $\mathbb{R}^{n}$ and $\left[\mathbf{0}, \boldsymbol{L}_{\mathrm{dom}}\right]$ :

$$
\int_{\mathbb{R}^{n}}(f g)(\boldsymbol{x}) d \boldsymbol{x}=\int_{0}^{L_{\mathrm{dom}}}(f g)(\boldsymbol{x}) d \boldsymbol{x}=\langle f g\rangle_{\boldsymbol{x}} \int_{0}^{L_{\mathrm{dom}}} d \boldsymbol{x}
$$

Furthermore, if we work in the context of a model with finite resolution, we set the signal to zero above the Nyquist wavenumber: $\left(\widehat{f} \widehat{g}^{*}\right)\left(\|\boldsymbol{k}\|>\left\|\boldsymbol{k}_{\boldsymbol{N}}\right\|\right)=\mathbf{0}$. Additionally, since $(f)$ and $(g)$ have zero $\boldsymbol{x}$-average, $(\widehat{f})(\boldsymbol{k}=\mathbf{0})=\mathbf{0}$, $\left(\widehat{g}^{*}\right)(\boldsymbol{k}=0)=0$, so $\left(\widehat{f} \widehat{g}^{*}\right)(\boldsymbol{k}=0)=0$, and we can write:

$$
\int_{\mathbb{R}_{+}^{n}} \operatorname{Re}\left(\widehat{f} \widehat{\boldsymbol{g}}^{*}\right)(\boldsymbol{k}) d \boldsymbol{k}=\int_{\boldsymbol{k}_{0}}^{\boldsymbol{k}_{N}} \operatorname{Re}\left(\widehat{f} \widehat{\boldsymbol{g}}^{*}\right)(\boldsymbol{k}) d \boldsymbol{k}=\left\langle\operatorname{Re}\left(\widehat{f} \widehat{\boldsymbol{g}}^{*}\right)\right\rangle_{\boldsymbol{k}} \int_{\boldsymbol{k}_{0}}^{\boldsymbol{k}_{N}} d \boldsymbol{k}
$$

We conclude by using the result from step 3 :

$$
\begin{aligned}
& \int_{\mathbb{R}^{n}} f(\boldsymbol{x}) \boldsymbol{g}(\boldsymbol{x}) d \boldsymbol{x}=2 \int_{\mathbb{R}_{+}^{n}} d \boldsymbol{k} \cdot \operatorname{Re}\left[\widehat{f}(\boldsymbol{k}) \widehat{\boldsymbol{g}}(\boldsymbol{k})^{*}\right] \\
& \langle f \boldsymbol{g}\rangle_{\boldsymbol{x}} \int_{0}^{\boldsymbol{L}_{\mathrm{dom}}} d \boldsymbol{x}=2\left\langle\operatorname{Re}\left(\widehat{f} \widehat{\boldsymbol{g}}^{*}\right)\right\rangle_{\boldsymbol{k}} \int_{\boldsymbol{k}_{0}}^{\boldsymbol{k}_{\boldsymbol{N}}} d \boldsymbol{k}
\end{aligned}
$$

$$
\langle f g\rangle_{x}=\mathcal{N}\left\langle\operatorname{Re}\left(\widehat{f} \widehat{g}^{*}\right)\right\rangle_{k} \mid \mathcal{N} \stackrel{\text { def }}{=} 2 \int_{k_{0}}^{k_{N}} d \boldsymbol{k} / \int_{0}^{L_{\mathrm{dom}}} d \boldsymbol{x}
$$

which proves identity 32

We are now ready to apply the identity 32 to the moist static energy spatial variance budget terms (e.g. Wing and Emanuel [2014], Andersen and Kuang [2012]). The budget considers zonal deviations of physical variables $X$, defined as:

$$
X^{\prime} \stackrel{\text { def }}{=} X-\langle X\rangle_{x}
$$

Both $X^{\prime}$ and $Y^{\prime}$ are doubly-periodic on $\left[0, L_{\text {dom }}\right]$ and have zero $\boldsymbol{x}$-average, which allows us to use the identity 32 First, by applying the identity 32 to $f=g=H^{\prime}$, we see that the spectral-mean of the spectrum $\varphi$ is proportional to the spatial variance of $H, \operatorname{var} H \stackrel{\text { def }}{=}\left\langle H^{\prime} H^{\prime}\right\rangle_{x}$ :

$$
\langle\varphi\rangle_{k}=\mathcal{N} \cdot \operatorname{var} H .
$$

By taking a logarithmic time-derivative of identity 32 we see that if normalized, the spectral-mean evolution of the spectrum $\varphi$ equals the evolution of $\operatorname{var} H$ :

$$
\begin{aligned}
\frac{1}{\langle\varphi\rangle_{k}}\left\langle\frac{\partial \varphi}{\partial t}\right\rangle_{k} & =\frac{1}{\operatorname{var} H} \frac{\partial(\operatorname{var} H)}{\partial t}, \\
\sum_{i=\mathrm{l}, \mathrm{sw}, \mathrm{sf}, \mathrm{adv}} \frac{\left\langle\dot{\varphi}_{i}\right\rangle_{k}}{\langle\varphi\rangle_{k}} & =\sum_{i=\mathrm{lw}, \mathrm{sw}, \mathrm{sf}, \mathrm{adv}} \frac{(\operatorname{var} H)_{i}}{\operatorname{var} H},
\end{aligned}
$$




$$
\sum_{i=I \mathrm{w}, \mathrm{sw}, \mathrm{sf}, \mathrm{adv}} \frac{\left\langle\operatorname{Re}\left\{\widehat{H}^{*} \widehat{\dot{H}}_{i}\right\}\right\rangle_{\boldsymbol{k}}}{\langle\varphi\rangle_{\boldsymbol{k}}}=\sum_{i=\mathrm{ww}, \mathrm{sw}, \mathrm{sf}, \mathrm{adv}} \frac{\left\langle H^{\prime} \dot{H}_{i}^{\prime}\right\rangle_{\boldsymbol{x}}}{\operatorname{var} H}
$$

This means that the physical understanding of how each process contributes to var $H$ (e.g. sections 3.1-3.4 of Wing et al. [2017|) carries over to how each process contributes to the average of $\varphi$ in spectral space. individual tendency. For a given flux $i=\mathrm{lw}$, sw, sf, adv, we choose $f=\dot{H}_{i}$ and $g=H^{\prime}$; combining identity 32 to identity 43 shows that the normalized spectral coherence between MSE and flux (i) equals the normalized spatial correlation between MSE and flux (i):

$$
\forall i, \frac{\left\langle\operatorname{Re}\left\{\widehat{H}^{*} \widehat{\dot{H}}_{i}\right\}\right\rangle_{k}}{\langle\varphi\rangle_{k}}=\frac{\left\langle H^{\prime} \dot{H}_{i}^{\prime}\right\rangle_{\boldsymbol{x}}}{\operatorname{var} H}
$$




\section{SUMMARY TABLE FOR ALL EXPERIMENTS}

\begin{tabular}{|c|c|c|c|c|c|c|c|}
\hline $\operatorname{Var} \downarrow \operatorname{Exp} \rightarrow$ & CAM280 & CAM285 & CAM290 & CAM295 & CAM300 & CAM305 & CAM310 \\
\hline$(L)_{\mathrm{G} / \mathrm{M}}[1000 \mathrm{~km}]$ & $3.7 / 5.5$ & $2.8 / 4.6$ & $4.6 / 2.7$ & $2.2 / 2.5$ & $2.4 / 2.7$ & $2.0 / 1.8$ & $1.9 / 1.3$ \\
\hline$\left(\left\langle\dot{\varphi}_{i}\right\rangle /\langle\varphi\rangle\right)_{\mathrm{G}}\left[\mathrm{d}^{-1}\right]$ & $0.30 .60 .4-1.0$ & $0.30 .40 .4-0.8$ & $0.20 .20 .4-0.6$ & $0.30 .10 .4-0.6$ & $0.20 .10 .2-0.4$ & $0.20 .00 .2-0.3$ & $0.20 .00 .2-0.2$ \\
\hline$\left(\left\langle\dot{\varphi}_{i}\right\rangle /\langle\varphi\rangle\right)_{\mathrm{M}}\left[\mathrm{d}^{-1}\right]$ & $0.30 .4-0.0-0.7$ & $0.30 .2-0.0-0.5$ & $0.10 .1-0.0-0.3$ & $0.10 .1-0.1-0.1$ & $0.10 .1-0.0-0.1$ & $0.00 .0-0.0-0.0$ & $0.00 .0-0.00 .0$ \\
\hline$\left(10 \dot{L}_{i} / L\right)_{\mathrm{G}}\left[\mathrm{d}^{-1}\right]$ & $1.9-0.70 .2-0.1$ & $2.9-0.20 .4-2.2$ & $2.4-0.01 .0-2.4$ & $1.6-0.01 .0-1.9$ & $1.1-0.10 .5-0.8$ & $1.4-0.10 .8-1.4$ & $0.6-0.00 .6-0.5$ \\
\hline$\left(10 \dot{L}_{i} / L\right)_{\mathrm{M}}\left[\mathrm{d}^{-1}\right]$ & $-0.1-0.40 .00 .4$ & $0.8-0.20 .1-0.6$ & $0.8-0.00 .0-0.9$ & $0.2-0.0-0.0-0.2$ & $-0.0-0.0-0.30 .3$ & $0.1-0.0-0.10 .0$ & $0.0-0.0-0.00 .0$ \\
\hline$\left(\mathcal{L}_{i}\right)_{\mathrm{G}}[1000 \mathrm{~km}]$ & 4.23 .44 .13 .6 & 4.42 .62 .83 .1 & 7.54 .34 .95 .7 & 3.12 .12 .42 .6 & 3.12 .12 .42 .5 & 2.71 .52 .32 .5 & 2.21 .62 .12 .1 \\
\hline$\left(\mathcal{L}_{i}\right)_{\mathrm{M}}[1000 \mathrm{~km}]$ & 5.35 .05 .65 .2 & 5.84 .24 .44 .9 & 4.42 .61 .63 .7 & 3.32 .42 .63 .0 & 2.62 .55 .91 .8 & 2.21 .62 .81 .8 & 1.51 .31 .41 .6 \\
\hline $\operatorname{Var} \downarrow \operatorname{Exp} \rightarrow$ & RRTM280 & RRTM285 & RRTM290 & RRTM295 & RRTM300 & RRTM305 & RRTM310 \\
\hline$(L)_{\mathrm{G} / \mathrm{M}}[1000 \mathrm{~km}]$ & $2.6 / 4.4$ & $1.7 / 1.5$ & $1.5 / 1.5$ & $2.2 / 1.8$ & $2.1 / 1.7$ & $1.5 / 1.4$ & $1.5 / 2.0$ \\
\hline$\left(\left\langle\dot{\varphi}_{i}\right\rangle /\langle\varphi\rangle\right)_{\mathrm{G}}\left[\mathrm{d}^{-1}\right]$ & $0.30 .40 .5-1.0$ & $0.30 .30 .5-0.8$ & $0.30 .10 .5-0.8$ & $0.20 .10 .3-0.4$ & $0.20 .00 .2-0.3$ & $0.20 .00 .1-0.1$ & $0.10 .00 .2-0.1$ \\
\hline$\left(\left\langle\dot{\varphi}_{i}\right\rangle /\langle\varphi\rangle\right)_{\mathrm{M}}\left[\mathrm{d}^{-1}\right]$ & $0.20 .3-0.1-0.4$ & $-0.00 .2-0.1-0.1$ & $0.00 .1-0.1-0.1$ & $0.00 .1-0.1-0.0$ & $0.00 .0-0.0-0.1$ & $0.00 .0-0.0-0.0$ & $0.00 .0-0.0-0.0$ \\
\hline$\left(10 \dot{L}_{i} / L\right)_{\mathrm{G}}\left[\mathrm{d}^{-1}\right]$ & $2.3-0.60 .2-0.8$ & $2.1-0.4-0.6-0.3$ & $1.9-0.20 .3-1.4$ & $2.0-0.11 .1-2.4$ & $0.7-0.10 .3-0.4$ & $0.8-0.10 .6-0.7$ & $0.8-0.10 .6-0.8$ \\
\hline$\left(10 \dot{L}_{i} / L\right)_{\mathrm{M}}\left[\mathrm{d}^{-1}\right]$ & $0.2-0.30 .3-0.3$ & $0.5-0.10 .0-0.4$ & $0.5-0.10 .1-0.7$ & $0.2-0.0-0.1-0.0$ & $0.1-0.0-0.10 .0$ & $0.1-0.0-0.20 .0$ & $0.0-0.0-0.10 .2$ \\
\hline$\left(\mathcal{L}_{i}\right)_{\mathrm{G}}[1000 \mathrm{~km}]$ & 3.42 .42 .92 .7 & 2.41 .51 .31 .6 & 2.21 .31 .41 .6 & 3.51 .82 .53 .0 & 2.61 .72 .02 .1 & 2.20 .91 .92 .1 & $2.3-0.02 .02 .5$ \\
\hline$\left(\mathcal{L}_{i}\right)_{\mathrm{M}}[1000 \mathrm{~km}]$ & 4.83 .92 .34 .6 & -20.21 .41 .52 .4 & 3.71 .41 .22 .9 & 2.71 .72 .12 .4 & 2.31 .62 .31 .7 & 1.91 .33 .11 .3 & $2.41 .72 .6-22.8$ \\
\hline
\end{tabular}

\begin{tabular}{|c|c|c|c|c|c|c|}
\hline $\operatorname{Var} \downarrow$ Exp $\rightarrow$ & MD RAD SFC & MD RAD & MD SFC & SQ CAM & SQ RRTM & BSQ RRTM \\
\hline$(L)_{\mathrm{G} / \mathrm{M}}[1000 \mathrm{~km}]$ & $2.2 / 2.3$ & $2.2 / 2.3$ & $2.1 / 4.8$ & $0.4 / 0.8$ & $0.4 / 0.7$ & $0.5 / 1.1$ \\
\hline$\left(\left\langle\dot{\varphi}_{i}\right\rangle /\langle\varphi\rangle\right)_{\mathrm{G}}\left[\mathrm{d}^{-1}\right]$ & $0.00 .00 .0-0.3$ & $0.00 .00 .4-0.7$ & $0.20 .10 .0-0.1$ & $0.10 .00 .1-0.1$ & $0.10 .00 .1-0.1$ & $0.10 .00 .1-0.1$ \\
\hline$\left(\left\langle\dot{\varphi}_{i}\right\rangle /\langle\varphi)_{\mathrm{M}}\left[\mathrm{d}^{-1}\right]\right.$ & $0.00 .00 .0-0.4$ & $0.00 .00 .4-0.8$ & $0.10 .00 .0-0.1$ & $0.00 .0-0.0-0.1$ & $0.00 .0-0.0-0.1$ & $0.00 .0-0.1-0.0$ \\
\hline$\left(10 \dot{L}_{i} / L\right)_{\mathrm{G}}\left[\mathrm{d}^{-1}\right]$ & $0.00 .00 .0-0.8$ & $0.00 .01 .7-2.6$ & $0.4-0.10 .00 .5$ & $0.5-0.10 .30 .1$ & $0.3-0.10 .20 .3$ & $0.2-0.10 .30 .3$ \\
\hline$\left(10 \dot{L}_{i} / L\right)_{\mathrm{M}}\left[\mathrm{d}^{-1}\right]$ & $0.00 .00 .0-1.2$ & $0.00 .01 .2-2.5$ & $0.1-0.10 .00 .2$ & $0.0-0.00 .0-0.0$ & $0.1-0.00 .1-0.0$ & $-0.0-0.0-0.10 .3$ \\
\hline$\left(\mathcal{L}_{i}\right)_{\mathrm{G}}[1000 \mathrm{~km}]$ & N/A N/A N/A 3.3 & N/A N/A 3.0 3.1 & 2.31 .8 N/A 1.1 & 0.50 .40 .40 .3 & 0.40 .30 .40 .2 & 0.60 .40 .50 .3 \\
\hline$\left(\mathcal{L}_{i}\right)_{\mathrm{M}}[1000 \mathrm{~km}]$ & N/A N/A N/A 3.2 & N/A N/A 3.0 3.0 & 5.33 .7 N/A 3.9 & 0.80 .70 .60 .8 & 0.80 .70 .60 .7 & 1.01 .01 .20 .0 \\
\hline & & & & & & \\
\hline
\end{tabular}

TA B LE 4 For each experiment (Exp), variables (Var) from top to bottom are: Time-averaged convective aggregation scale $L$ (in $1000 \mathrm{~km}$ ) during the growth phase (subscript G) and the mature phase (subscript M), time-averaged aggregation rate during the growth phase $\left(\left\langle\dot{\varphi}_{i}\right\rangle /\langle\varphi\rangle_{G}\right.$ (in day $\left.{ }^{-1}\right)$, time-averaged aggregation rate during the mature phase $\left(\left\langle\dot{\varphi}_{i}\right\rangle /\langle\varphi\rangle\right)_{M}\left(\right.$ in day $\left.{ }^{-1}\right), 10$ times the time-averaged expansion rate during the growth phase $\left(\dot{L}_{i} / L\right)_{G}$ (in day $\left.^{-1}\right)$, 10 times the time-averaged expansion rate during the mature phase $\left(L_{i} / L\right)_{M}$ (in day ${ }^{-1}$ ), time-averaged diabatic length scale during the growth phase $\left(\mathcal{L}_{i}\right)_{G}$ (in $1000 \mathrm{~km}$ ), and time-averaged diabatic length scale during the mature phase $\left(\mathcal{L}_{i}\right)_{M}$ (in $1000 \mathrm{~km}$ ). $i$ represents a generic flux in the legend; in practice, fluxes are listed in the following order: Longwave Shortwave Surface enthalpy flux Advective flux. 

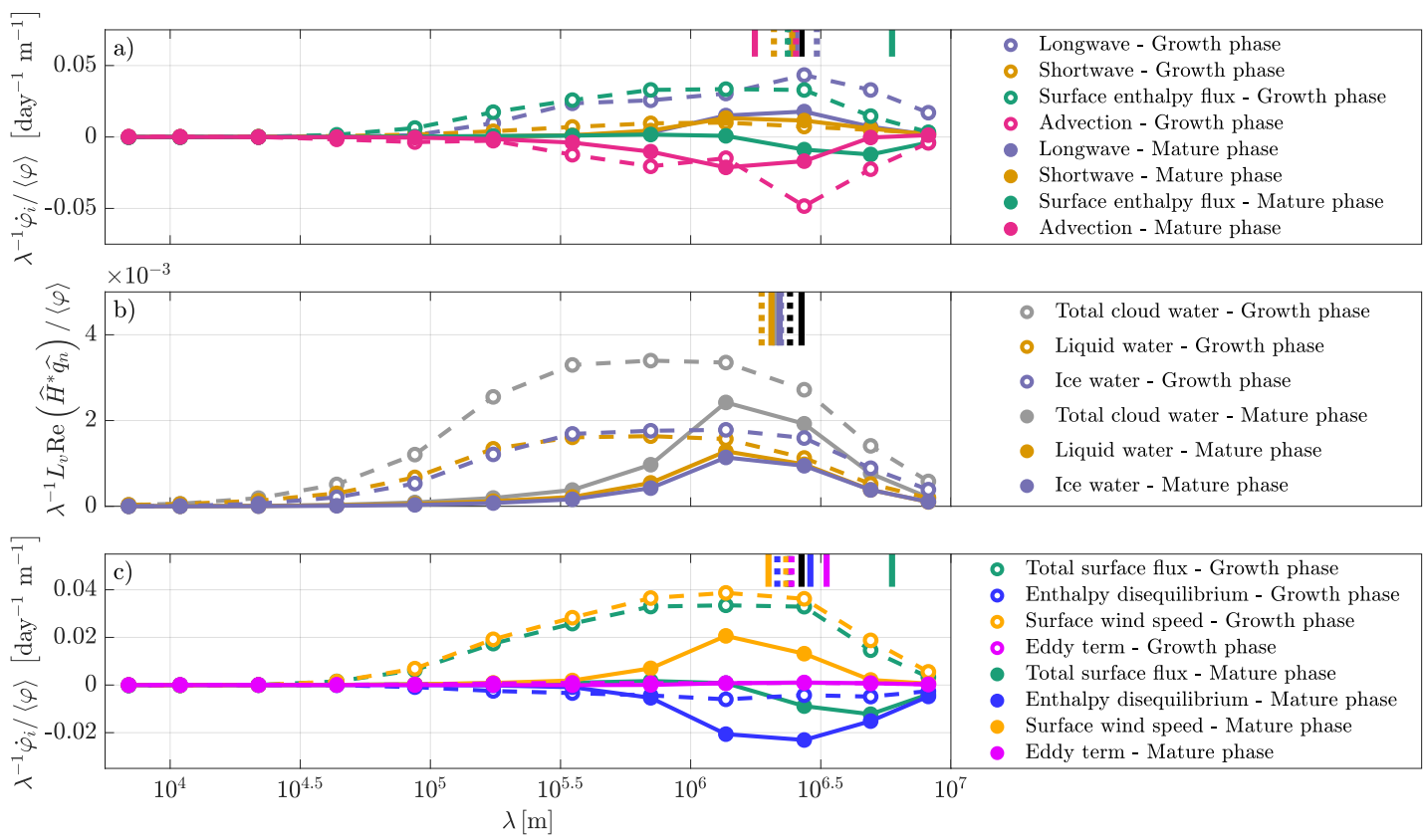

FI GURE 11 (a) Normalized power spectral tendencies (in day/m) of the longwave (blue), shortwave (orange), surface enthalpy (green) and advective (pink) fluxes of MSE during the growth (first month of simulation) and mature (last months of simulation) phases of the reference LC300CAM simulation. (b) Normalized spectral coherence (unitless) between MSE and cloud water concentration: Total cloud water (gray), liquid (orange) and ice (blue) water. (c) Normalized power spectral tendencies (in day $/ \mathrm{m}$ ) of the total surface enthalpy flux (green), enthalpy disequilibrium component (blue), surface wind speed component (orange) and eddy term (pink). The total normalization factor used in all three panels is equal to $\left(\lambda\langle\varphi\rangle \int_{k_{0}}^{k_{N}} d k\right)^{-1}$.

All spectra are octave-averaged (dots) and plotted versus wavelength (in $\mathrm{m}$ ) on a logarithmic scale. The diabatic length scale $\mathcal{L}_{i}$ (in $\mathrm{m}$ ) corresponding to each flux is indicated with a dotted vertical line (growth phase) and a full line (mature phase). For reference, the convective aggregation length scale $L$ (in $\mathrm{m}$ ) is depicted with a dotted black line (growth phase) and a full black line (mature phase).

In this appendix, we compare the distribution of the MSE power spectral tendencies $\dot{\varphi}_{i}$ across wavelengths $\lambda$ (Figure 113) to the spectral coherence of cloud water (Figure 11p) and the spectral tendencies $\dot{\varphi}_{i}$ of the surface enthalpy flux components listed in equation 19 (Figure 11 F). Following definition 6 the tendencies are normalized using the wavenumber-averaged MSE power spectrum $\langle\varphi\rangle$, the inverse wavelength $\lambda^{-1}$ and a wavenumber pre-factor, so that the spectra can be visually integrated over the logarithmic scale to yield the aggregation rate (in s ${ }^{-1}$ ):

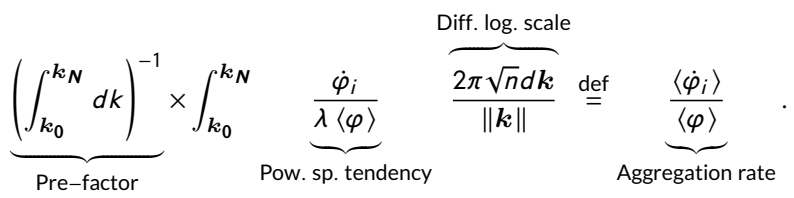


The cloud water spectral coherence with MSE, depicted in Figure 11p, is defined analogously to the MSE spectral tendency $\dot{\varphi}_{i}$ : In equation 11 we substitute the spatial Fourier transform of the cloud water path $\left(\mathrm{in} \mathrm{kg} / \mathrm{m}^{2}\right)$ for the spatial Fourier transform $\hat{\dot{H}}_{i}$ of the MSE flux $\{i\}$ (in W/m²). We then multiply the cloud coherence by the latent heat of vaporization of water vapor $L_{v}$ (in units $\mathrm{J} \mathrm{kg}^{-1}$, evaluated using standard atmospheric temperature conditions) to make it unitless.

The diabatic length scale $\mathcal{L}_{i}$ of each flux $\{i\}$ is the centroid of the dots, and is indicated with a vertical line in the upper part of each panel. During the first month of the simulation:

- The longwave flux operates at the largest scale, larger than the MSE anomaly scale.

- The MSE anomaly scale is very close to the MSE advection scale as convection aggregates.

- The shortwave flux operates at the smallest scale, below the MSE anomaly scale, resulting in the shortwave shrinking effect.

- The cloud water coherence spectrum shows that ice prevails over liquid water at scales larger than $~ 300 \mathrm{~km}$, consistent with the longwave flux adding MSE power at larger scales than the shortwave flux.

- The surface enthalpy flux operates at a larger then smaller scale than the MSE anomaly scale. From the decomposition of the surface enthalpy flux during the first month, the shape of the surface enthalpy flux is mostly determined by its surface wind component, with a broad peak between $\sim 1000 \mathrm{~km}$ and $\sim 3000 \mathrm{~km}$.

In contrast, during the last months of the simulation:

- The longwave and shortwave fluxes operate at scales closer to the MSE anomaly scale, and the tendencies are more peaked, between $\sim 1000 \mathrm{~km}$ and $\sim 5000 \mathrm{~km}$.

- The ice and liquid water spectra are also more similar to each other, as well as more peaked.

- The advective flux removes MSE power at all scales and peaks below the MSE anomaly scale. Therefore, it preferentially removes power at smaller scales, homogenizing small scale MSE anomalies faster than large-scale ones. This explains the stretching effect of advection in the reference LC300CAM case.

- The surface enthalpy fluxes add MSE power through the surface wind effect up to $2000 \mathrm{~km}$ and remove energy through the enthalpy disequilibrium effect above that scale. As a result, the diabatic length scale is artificially large because the surface enthalpy flux aggregation tendency $\left\langle\dot{\varphi}_{\text {sf }}\right\rangle$ is double-signed. It is then easier to think of the single-signed components of the surface enthalpy flux: the surface wind component adds power with a peaked spectrum centered around $\sim 2000 \mathrm{~km}$, while the surface enthalpy disequilibrium component removes power with a broader spectrum centered around $\sim 3000 \mathrm{~km}$.

\section{ENDNOTES}

\section{REFERENCES}

Andersen, J. A. and Kuang, Z. (2012) Moist static energy budget of MJO-like disturbances in the atmosphere of a zonally sym1. metric aquaplanet. Journal of Climate, 25, 2782-2804. URL:http://journals . ametsoc . org/doi/abs/10.1175/JCLI-D-1100168.1 .

Arnold, N. (2015) Convective self-aggregation and the Madden-Julian oscillation. URL: http://kiwi.atmos.colostate.edu/ cmmap/research/docs/aug15/talknarnold.pdf. 
Arnold, N. and Putman, W. (2018) Non-rotating convective self-aggregation in a limited area AGCM. Journal of Advances in Modeling Earth Systems.

Arnold, N. P. and Randall, D. A. (2015) Global-scale convective aggregation: Implications for the Madden-Julian Oscillation. Journal of Advances in Modeling Earth Systems, 7, 1499-1518. URL: http://doi . wiley . com/10.1002/2015MS000498

Bretherton, C. S., Blossey, P. N. and Khairoutdinov, M. (2005) An Energy-Balance Analysis of Deep Convective Self-Aggregation above Uniform SST. Journal of the Atmospheric Sciences, 62, 4273-4292.

Bretherton, C. S. and Khairoutdinov, M. F. (2015) Convective self-aggregation feedbacks in near-global cloud-resolving simulations of an aquaplanet. Journal of Advances in Modeling Earth Systems, 7, 1765-1787. URL: http://doi.wiley.com/10. 1002/2015MS000499.

Collins, W. D., Bitz, C. M., Blackmon, M. L., Bonan, G. B., Bretherton, C. S., Carton, J. A., Chang, P., Doney, S. C., Hack, J. J., Henderson, T. B., Kiehl, J. T., Large, W. G., McKenna, D. S., Santer, B. D. and Smith, R. D. (2006) The Community Climate

System Model version 3 (CCSM3). Journal of Climate, 19, 2122-2143. URL: http://journals.ametsoc.org/doi/abs/10. $1175 /$ JCLI3761.1.

Coppin, D. and Bony, S. (2015) Physical mechanisms controlling the initiation of convective self-aggregation in a general circulation model. Journal of Advances in Modeling Earth Systems, n/a-n/a. URL: http://doi . wiley . com/10.1002/2015MS000571.

- (2017) Internal variability in a coupled General Circulation Model in Radiative-Convective Equilibrium. Geophysical Research Letters. URL:http://doi.wiley.com/10.1002/2017GL073658.

Dunn, P. (2005) Measurement and Data Analysis for Engineering and Science. CRC Press, Taylor \& Francis Group, Boca Raton FL. URL: https://www .taylorfrancis.com/books/9781466595033citeulike-article-id: 9782805

Frigo, M. and Johnson, S. G. (2005) The design and implementation of FFTW3. In Proceedings of the IEEE, vol. 93, 216-231. URL: http://www.fftw.org/fftw-paper-ieee.pdf.

Grabowski, W. W. and Moncrieff, M. W. (2004) Moisture-convection feedback in the tropics. Quarterly Journal of the Royal Meteorological Society, 130, 3081-3104. URL:http://doi . wiley.com/10.1256/qj.03.135.

Held, I. M., Hemler, R. S. and Ramaswamy, V. (1993) Radiative-Convective Equilibrium with Explicit Two-Dimensional Moist Convection.

Held, I. M., Zhao, M. and Wyman, B. (2007) Dynamic Radiative-Convective Equilibria Using GCM Column Physics. Journal of the Atmospheric Sciences, 64, 228-238. URL: http://journals . ametsoc . org/doi/abs/10.1175/JAS3825.11.

Hohenegger, C. and Stevens, B. (2018) The role of the permanent wilting point in controlling the spatial distribution of precipitation. Proceedings of the National Academy of Sciences, 115, 5692-5697. URL: http://www.ncbi.

nlm.nih.gov/pubmed/29760083http://www.pubmedcentral.nih.gov/articlerender.fcgi?artid=PMC5984498http: //www.pnas.org/lookup/doi/10.1073/pnas.1718842115.

Holloway, C. E. and Woolnough, S. J. (2016) The sensitivity of convective aggregation to diabatic processes in idealized radiative-convective equilibrium simulations. Journal of Advances in Modeling Earth Systems, 8, 166-195. URL: http: //doi.wiley.com/10.1002/2015MS000511

Houze, R. A. (1989) Observed structure of mesoscale convective systems and implications for large-scale heating. Quarterly Journal of the Royal Meteorological Society, 115, 425-461. URL: http://doi .wiley . com/10.1002/qj .49711548702.

Iacono, M. J., Delamere, J. S., Mlawer, E. J., Shephard, M. W., Clough, S. A. and Collins, W. D. (2008) Radiative forcing by longlived greenhouse gases: Calculations with the AER radia- tive transfer models. Journal of Geophysical Research Atmospheres, 113. URL: http://onlinelibrary.wiley.com/doi/10.1029/2008JD009944/full.

Khairoutdinov, M. F. and Randall, D. a. (2003) Cloud Resolving Modeling of the ARM Summer 1997 IOP: Model Formulation, Results, Uncertainties, and Sensitivities. Journal of the Atmospheric Sciences, 60, 607-625. 
Muller, C. and Bony, S. (2015) What favors convective aggregation and why? Geophysical Research Letters, 42, 5626-5634. URL: http://doi.wiley.com/10.1002/2015GL064260

Muller, C. J. and Held, I. M. (2012) Detailed Investigation of the Self-Aggregation of Convection in Cloud-Resolving Simula1. tions. Journal of the Atmospheric Sciences, 69, 2551-2565. URL: http://journals . ametsoc. org/doi/abs/10.1175/JAS-D$11-0257.1$

Posselt, D. J., Van Den Heever, S., Stephens, G. and Igel, M. R. (2012) Changes in the interaction between tropical convection, radiation, and the large-scale circulation in a warming environment. Journal of Climate, 25, 557-571. URL: http://journals.ametsoc.org/doi/abs/10.1175/2011 JCLI4167.1.

Ramanathan, V. and Coakley, J. A. (1978) Climate modeling through radiative-convective models. Reviews of Geophysics, 16, 465-489. URL:http://doi.wiley.com/10.1029/RG016i004p00465.

Tompkins, A. and Craig, G. C. (1998) Radiative-convective equilibrium in a three-dimensional cloud-ensemble model. Quarterly Journal of the Royal Meteorological Society, 124, 2073-2097.

Vallis, G. K. (2006) Athmospheric and oceanic fluid dynamics, Fundamental and large-scale circulation.

Wing, A. A., Camargo, S. J. and Sobel, A. H. (2016) Role of radiative-convective feedbacks in spontaneous tropical cyclogenesis 1. in idealized numerical simulations. Journal of the Atmospheric Sciences, 73, JAS-D-15-0380.1. URL: http://journals ametsoc.org/doi/10.1175/JAS-D-15-0380.1

Wing, A. A. and Cronin, T. W. (2016) Self-aggregation of convection in long channel geometry. Quarterly Journal of the Royal Meteorological Society, 142, 1-15. URL:http://doi.wiley.com/10.1002/qj . 2628.

Wing, A. A., Emanuel, K., Holloway, C. E. and Muller, C. (2017) Convective Self-Aggregation in Numerical Simulations: A Review. Surveys in Geophysics. URL: http://link.springer.com/10.1007/s10712-017-9408-4

Wing, A. a. and Emanuel, K. a. (2014) Physical mechanisms controlling self-aggregation of convection in idealized numerin cal modeling simulations. Journal of Advances in Modeling Earth Systems, 5, 59-74. URL: http://dx.doi.org/10.1002/ 2013MS000269

Yang, D. (2017) Boundary layer height and buoyancy determine the horizontal scale of convective self-aggregation. Journal of the Atmospheric Sciences, JAS-D-17-0150.1. URL: http://journals . ametsoc . org/doi/10.1175/JAS-D-17-0150. 1

- (2018) Boundary Layer Diabatic Processes, the Virtual Effect, and Convective Self-Aggregation. Journal of Advances in Modeling Earth Systems, 10, 2163-2176. URL:http://doi . wiley.com/10.1029/2017MS001261

Zhang, C. (2005) Madden-Julian Oscillation. URL:http://doi .wiley.com/10.1029/2004RG000158. 